\title{
Limit-Cycle Oscillations in Unsteady Flows Dominated by Intermittent Leading-Edge Vortex Shedding
}

\author{
Kiran Ramesh ${ }^{\mathrm{a}, *}$, Joseba Murua ${ }^{\mathrm{c}}$, Ashok Gopalarathnam ${ }^{\mathrm{b}}$ \\ ${ }^{a}$ Aerospace Sciences Division, School of Engineering, University of Glasgow, Glasgow, \\ G12 $8 Q Q, U K$ \\ ${ }^{b}$ Department of Mechanical and Aerospace Engineering, North Carolina State University, \\ Raleigh, NC 27695-7910, USA \\ ${ }^{c}$ Department of Mechanical Engineering Sciences, University of Surrey, Guildford, GU2 \\ $7 X H, U K$
}

\section{Abstract}

High-frequency limit-cycle oscillations of an airfoil at low Reynolds number are studied numerically. This regime is characterized by large apparentmass effects and intermittent shedding of leading-edge vortices. Under these conditions, leading-edge vortex shedding has been shown to result in favorable consequences such as high lift and efficiencies in propulsion/power extraction, thus motivating this study. The aerodynamic model used in the aeroelastic framework is a potential-flow-based discrete-vortex method, augmented with intermittent leading-edge vortex shedding based on a leadingedge suction parameter reaching a critical value. This model has been validated extensively in the regime under consideration and is computationally cheap in comparison with Navier-Stokes solvers. The structural model used has degrees of freedom in pitch and plunge, and allows for large amplitudes and cubic stiffening. The aeroelastic framework developed in this paper is employed to undertake parametric studies which evaluate the impact of different types of nonlinearity. Structural configurations with pitch-to-plunge frequency ratios close to unity are considered, where the flutter speeds are lowest (ideal for power generation) and reduced frequencies are highest. The range of reduced frequencies studied is two to three times higher than most airfoil studies, a virtually unexplored regime. Aerodynamic nonlinearity re-

\footnotetext{
${ }^{*}$ Corresponding author

Email addresses: kiran.ramesh@glasgow.ac.uk (Kiran Ramesh), j.murua@surrey.ac.uk (Joseba Murua), agopalar@ncsu.edu (Ashok Gopalarathnam)
} 
sulting from intermittent leading-edge vortex shedding always causes a supercritical Hopf bifurcation, where limit-cycle oscillations occur at freestream velocities greater than the linear flutter speed. The variations in amplitude and frequency of limit-cycle oscillations as functions of aerodynamic and structural parameters are presented through the parametric studies. The excellent accuracy/cost balance offered by the methodology presented in this paper suggests that it could be successfully employed to investigate optimum setups for power harvesting in the low-Reynolds-number regime.

\section{Nomenclature}

$\alpha$

$\bar{\omega}=\omega_{h} / \omega_{\alpha}$

$\beta_{\alpha}$

$\beta_{h}$

$\boldsymbol{q}=\left[\begin{array}{ll}h & \alpha\end{array}\right]^{T}$

$\gamma(\theta)$

$\kappa=\pi \rho c^{2} / 4 m$

$\omega=2 \pi / T$

$\omega_{\alpha}=\sqrt{k_{\alpha} / I_{\alpha}}$

$\omega_{h}=\sqrt{k_{h} / m}$

$\theta$

$A_{0}, A_{1}, A_{2} \ldots$

$c$

$C_{d}$

$C_{l}$

$C_{m}$ pitch angle

frequency ratio

coefficient defining cubic stiffening in pitch

coefficient of cubic stiffening in plunge

generalized coordinates

chordwise vorticity on airfoil

inverse mass ratio

angular frequency of sinusoidal motion

characteristic frequency of pitch mode

characteristic frequency of plunge mode

variable of transformation of chordwise distance

Fourier coefficients

airfoil chord

drag coefficient, per unit span

lift coefficient, per unit span

pitching moment coefficient, per unit span 


\begin{tabular}{|c|c|}
\hline$h$ & plunge displacement \\
\hline$I_{\alpha}$ & airfoil moment of inertia about pivot \\
\hline$k=\omega c / 2 U$ & reduced frequency \\
\hline$k_{\alpha}$ & linear pitch stiffness, per unit span \\
\hline$k_{h}$ & linear plunge stiffness, per unit span \\
\hline$L E S P$ & leading edge suction parameter \\
\hline$m$ & mass of airfoil \\
\hline$r_{\alpha}=2 \sqrt{I_{\alpha} / m c^{2}}$ & airfoil radius of gyration about pivot \\
\hline$S$ & measure of suction at the leading edge \\
\hline$S_{\alpha}$ & static moment of airfoil about pivot, per unit span \\
\hline$T$ & time period for sinusoidal motions \\
\hline$t$ & physical time \\
\hline$t^{*}=t U / c$ & non-dimensional time \\
\hline$U$ & freestream velocity \\
\hline$U^{*}=U / \omega_{\alpha} c$ & nondimensional velocity \\
\hline$U_{F}$ & linear flutter velocity \\
\hline$W(\theta)$ & induced velocity normal to airfoil \\
\hline$x_{\alpha}=2 S_{\alpha} / m c$ & $\begin{array}{l}\text { distance of center of gravity aft of pivot, nondimension- } \\
\text { alized by } c\end{array}$ \\
\hline$x_{p}$ & $\begin{array}{l}\text { distance of pivot aft of airfoil leading edge, nondimen- } \\
\text { sionalized by } c\end{array}$ \\
\hline$x_{C G}$ & $\begin{array}{l}\text { distance of center of gravity aft of pivot nondimension- } \\
\text { alized by } c\end{array}$ \\
\hline DOF & degrees of freedom \\
\hline
\end{tabular}




\section{Introduction}

Fluid-structure interaction often leads to undesirable consequences such as divergence, control reversal, and flutter [1,2], but it has also been shown to be beneficial in animal flight and swimming [3, 4, 5], for instance. These potential benefits have generated interest amongst the micro air vehicle (MAV) community, who aim to take inspiration from nature in designing flapping flyers in small sizes for low speeds [6, 7, 8]. Aeroelastic phenomena have also been employed successfully in novel energy-harvesting methods $[9,10,11,12,13]$, whereby nonlinear aeroelastic effects are exploited to extract energy from the incoming flow.

The objective of this research is to investigate nonlinear aeroelasticity in the high-reduced-frequency, low-Reynolds-number regime. Linear aeroelastic theory, such as that developed by Theodorsen $[14,15]$ can predict the freestream velocity above which the system becomes unstable and the airfoil oscillations grow exponentially. The presence of nonlinearities in the system, however, affects not only the flutter speed but also the characteristics of the system response. These nonlinearities could be either aerodynamic or structural and often result in constant-amplitude, stable vibrations. In fact, such non-destructive limit-cycle oscillations (LCOs) are the basis of the passive power-generation methods mentioned earlier.

Structural nonlinearities may arise owing to large deformations, material properties, or loose linkages [16]. The effects of structural nonlinearities on airfoil aeroelasticity have been studied by several authors, focusing on different types of nonlinear spring behavior such as bilinear or cubic variation in stiffness (see Refs. [17, 18]). A comprehensive review of such studies is given by Lee et al. [16]. These studies assume that the aerodynamics are linear, that is, the flow is incompressible, inviscid and attached to the airfoil. The onset and type of bifurcation, and amplitude and frequency of limit-cycle oscillations were investigated. Hard springs (positive cubic stiffening) result 
in a supercritical Hopf bifurcation, where LCOs occur only at freestream velocities greater than the linear flutter velocity and are independent of initial conditions. Soft springs (negative cubic stiffening), on the other hand, result in a subcritical Hopf bifurcation where LCOs may arise at velocities below the linear flutter velocity, depending on initial conditions. Further, chaotic oscillations are observed in a range of freestream velocities for some configurations.

Aerodynamic nonlinearities may result from compressibility or viscous effects [16]. Limit-cycle oscillations resulting from nonlinear aerodynamics due to compressibility effects (transonic flows) have been studied by Bendiksen [19]. Nonlinear aerodynamics caused by viscous flow phenomena are largely dependent on the Reynolds number and the reduced frequencies involved, and leading-edge vortices (LEVs) have been seen to play a crucial role. In helicopter and wind-turbine applications, which are necessarily associated with large Reynolds numbers and low reduced frequencies, LEVs and the resulting dynamic stall phenomenon might lead to violent vibrations and mechanical failure [20]. On the other hand, LEVs in high-frequency flows have been credited with contributing toward the success of high-lift flight in insects [21, 22, 23, 24], and high propulsive [25] and power-extraction [26] efficiencies.

In the dynamic-stall regime, Tang \& Dowell [27, 28] have studied flutter and forced response of a helicopter blade using the ONERA semi-empirical aerodynamic model developed by Tran \& Petot [29]. Sarkar \& Bijl [30] have published a study on the nonlinear aeroelastic behavior of an oscillating airfoil during dynamic stall, again with the ONERA model. In another study, Chantharasenawong [31] investigated aeroelastic response during dynamic stall using the Leishman-Beddoes semi-empirical aerodynamic model [32]. The limit-cycle oscillations in this regime were observed to be dependent on initial conditions.

Although the above semi-empirical models provide for quick computations, they rely on several parameters which need to be tuned with calibration data. Also, they can only be used in conditions that are bounded by validation with experimental data. Further, they merely provide estimations of the force coefficients without offering any physical insight into the aerodynamics involved. It is noted that, while there has been substantial research on aeroelasticity resulting from unsteady aerodynamics in the regimes associated with low-reduced-frequency helicopter dynamic stall, regimes of high reduced frequency and low Reynolds number associated with flapping wings 
and possibly power extraction have been relatively unexplored.

Nonlinear aeroelasticity can be modeled very accurately by combining high-fidelity computational fluid and structural solvers (see, for instance, Ref. [33]). Using such high-order computational methods, Poirel et al. [34] have studied limit-cycle oscillations caused by laminar separation bubbles at transitional Reynolds numbers, and Sváček et al. [35] have studied LCOs at high Reynolds numbers. Peng \& Zhu [36] have used a similar framework to assess energy extraction from oscillating structures. These methods offer greater insight into the flow physics than semi-empirical methods and are needed to validate low-order approaches based on approximations. They are, however, unsuitable for the study of a large parameter space or for use in design because of time and cost considerations.

Discrete-vortex methods can be used to model airfoil aerodynamics in the time-domain, at a lower computational cost than high-order CFD methods. Jones \& Platzer [37], for example, have analyzed airfoil flutter with a discretevortex method, although assuming attached flow on the airfoil. Flow separation and vortex formation can be modeled in discrete-vortex methods by shedding point vortices from the location of flow separation. A conventional limitation with these methods is that they assume flow separation (usually at the leading edge) at all times and do not define conditions at which it is initiated/terminated. Ramesh et al. [38] have developed a discrete-vortex aerodynamic method to model unsteady flows with intermittent LEV shedding using a leading-edge suction parameter (LESP). The unique aspect of this method is that vortex shedding is turned on or off at the leading edge using a criticality condition. This method is, therefore, ideally suited to modeling oscillatory airfoil flows in which intermittent LEV shedding is a key feature. In comparison with semi-empirical methods where several parameters are typically used, this model uses only a single empirical constant, the critical LESP, and is highly physics-based.

In this paper, the LESP-modulated discrete-vortex aerodynamic model is coupled with a two degree of freedom (2-DOF) nonlinear structural model in a effort to study fluid-structure interaction and limit-cycle oscillations in highfrequency, low-Reynolds-number, vortex-dominated flows. The aerodynamic and structural models employed are detailed in section 2 . In section 3 , these models are validated, in the regimes under consideration, against data from the literature. In section 4, the characteristics of LCOs in vortex-dominated flows as a function of relevant structural and aerodynamic parameters are presented. 


\section{Aeroelastic modeling}

A nonlinear aeroelastic model for a two-degree-of-freedom airfoil is presented in this section. The structural equations are geometrically nonlinear, accounting for large-amplitude motions, and cubic nonlinearities in the stiffness terms. For the aerodynamics, a discrete-vortex method with intermittent vortex shedding is employed. The resulting model, therefore, caters for nonlinearities in both structure and aerodynamics.

\subsection{Structural model: Geometrically nonlinear formulation of the two-degree- of-freedom airfoil}

The two-degree-of-freedom system subject to study is depicted in Figures 1 and 2. Figure 1 shows a rigid airfoil, elastically supported by translational and rotational springs in plunge, $h$, and pitch, $\alpha$. The corresponding generalized forces are lift, $L$, and pitching moment, $M$, respectively. The geometric parameters of the airfoil are illustrated in Figure 2. The pivot represents the point at which the springs are attached (often referred to as elastic axis as per 3D flexible-wing terminology), and the coordinate system employed for the derivation of the structural equations is fixed at this point in the undeformed configuration.

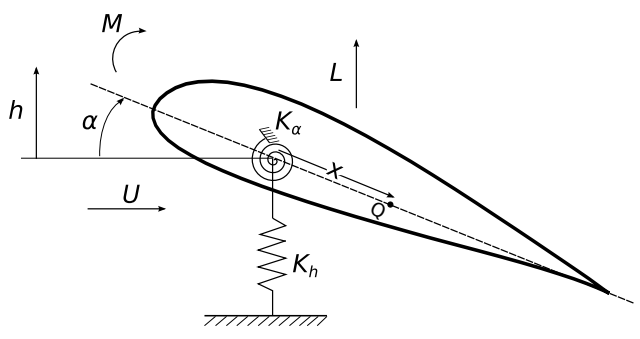

Figure 1: Airfoil degrees of freedom and lift and pitching moment acting on it.

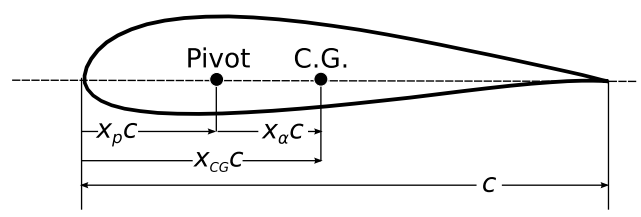

Figure 2: Locations of pivot and C.G. on the airfoil

From Figure 1, the horizontal $\left(\zeta_{x}\right)$ and vertical $\left(\zeta_{z}\right)$ displacements of a point $\mathrm{Q}$ on the airfoil chordline, at a distance $x$ from the pivot at its location in the undeformed configuration, are given by

$$
\begin{aligned}
\zeta_{x} & =x(1-\cos \alpha), \\
\zeta_{z} & =h-x \sin \alpha .
\end{aligned}
$$


If the mass per unit length at a point $x$ of the chordline is $\rho_{s}(x)$, then the kinetic, $T$, and potential, $U$, energies associated with the airfoil are obtained as

$$
\begin{aligned}
T & =\frac{1}{2} \int_{c} \rho_{s}\left(\dot{\zeta}_{x}^{2}+\dot{\zeta}_{z}^{2}\right) d x \\
& =\frac{1}{2} m \dot{h}^{2}-S_{\alpha} \cos \alpha \dot{h} \dot{\alpha}+\frac{1}{2} I_{\alpha} \dot{\alpha}^{2}, \\
U & =\int_{0}^{h} F_{h} d h+\int_{0}^{\alpha} F_{\alpha} d \alpha,
\end{aligned}
$$

where $(\dot{\bullet})$ indicates differentiation with respect to time, $m$ is the total mass of the airfoil, and $S_{\alpha}$ and $I_{\alpha}$ are its static and inertia moments about the pivot; $F_{h}=F_{h}(h)$ and $F_{\alpha}=F_{\alpha}(\alpha)$ are the restoring forces in plunge and pitch, respectively, and can include any spring nonlinearity such as cubic hardening/softening, bilinearity or hysteresis [16].

The kinetic and potential energies of the system, Eq. (2), must satisfy Lagrange's equations, which, neglecting structural damping read as

$$
\frac{d}{d t}\left(\frac{\partial T}{\partial \dot{\boldsymbol{q}}}\right)-\frac{\partial T}{\partial \boldsymbol{q}}+\frac{\partial U}{\partial \boldsymbol{q}}=\boldsymbol{Q},
$$

where the generalized coordinates and forces are, respectively, $\boldsymbol{q}=\left[\begin{array}{ll}h & \alpha\end{array}\right]^{T}$ and $\boldsymbol{Q}=\left[\begin{array}{ll}L & M\end{array}\right]^{T}$.

Applying Eq. (3) to the airfoil, the equations of motion are obtained as

$$
\begin{aligned}
m \ddot{h}-S_{\alpha} \ddot{\alpha} \cos \alpha+S_{\alpha} \dot{\alpha}^{2} \sin \alpha+F_{h} & =L, \\
-S_{\alpha} \cos \alpha \ddot{h}+I_{\alpha} \ddot{\alpha}+F_{\alpha} & =M,
\end{aligned}
$$

where the trigonometric functions account for geometric nonlinearities in the kinematics and arise from the definition of (nonlinear) airfoil displacements given in Eq. (1). In this work, only cubic springs are considered, and the forces $F_{h}(h)$ and $F_{\alpha}(\alpha)$, therefore, adopt the form of

$$
\begin{aligned}
& F_{h}(h)=k_{h} f_{h}(h)=k_{h}\left(h+\beta_{h} h^{3}\right), \\
& F_{\alpha}(\alpha)=k_{\alpha} f_{\alpha}(\alpha)=k_{\alpha}\left(\alpha+\beta_{\alpha} \alpha^{3}\right),
\end{aligned}
$$

where $k_{h}$ and $k_{\alpha}$ are the classical linear spring constants, and $\beta_{h}$ and $\beta_{\alpha}$ are constant coefficients for cubic stiffening with appropriate dimensions [16]. 
By multiplying Eq. (4) by $2 /(m c)$ and $4 /\left(m c^{2}\right)$ respectively, and assuming the cubic restoring loads given by Eq. (5), the nondimensional form of the equations of motion is obtained as

$$
\begin{aligned}
\frac{2}{c} \ddot{h}-x_{\alpha} \ddot{\alpha} \cos \alpha+x_{\alpha} \dot{\alpha}^{2} \sin \alpha+\frac{2}{c} \omega_{h}^{2} f_{h} & =\frac{4}{\pi} \kappa \frac{U^{2}}{c^{2}} C_{l} \\
-\frac{2}{c} x_{\alpha} \cos \alpha \ddot{h}+r_{\alpha}^{2} \ddot{\alpha}+\left(r_{\alpha} \omega_{\alpha}\right)^{2} f_{\alpha} & =\frac{8}{\pi} \kappa \frac{U^{2}}{c^{2}} C_{m}
\end{aligned}
$$

where the standard aeroelastic terminology has been used (see Nomenclature): $x_{\alpha}$ is the nondimensional static unbalance; $r_{\alpha}$ is the airfoil's radius of gyration referred to the pivot; $\kappa$ is the airfoil's inverse mass ratio; $\omega_{h}$ and $\omega_{\alpha}$ are the natural frequencies of the uncoupled plunging and pitching modes; and $C_{l}$ and $C_{m}$ are the lift and pitching moment coefficients.

The airfoil equations of motion are geometrically nonlinear, in both the kinematics and stiffness terms, capturing large-amplitude effects. It is worth mentioning that the kinematic nonlinearity has been frequently neglected in the literature when considering nonlinear stiffness terms (see, for instance, Lee et al. [16]); conversely, the spring nonlinearity is often neglected in geometrically nonlinear descriptions of the kinematics (e.g., Sváček et al. [35]). Note also that the linear (classical) version of the airfoil equations of motion [14] can be recovered by considering linear springs and assuming $\zeta_{x} \approx 0$ and $\zeta_{z} \approx h-x \alpha$ in the airfoil-displacement definition, Eq. (1).

\subsection{Aerodynamic model: Discrete-vortex model with intermittent LEV shed- ding}

The aerodynamic model used in the current work is a recently-developed discrete vortex method with a novel shedding criterion that modulates intermittent vortex shedding from the leading edge. The shedding criterion, governed by a maximum allowable leading-edge suction, is based on the critical value of a leading-edge suction parameter (LESP). This section briefly describes the main elements of the LESP-modulated discrete-vortex method (LDVM). The interested reader may refer to Refs. [38, 39] for further details.

\subsubsection{Large-angle unsteady thin-airfoil theory}

At the foundation of the LDVM is a large-angle unsteady thin-airfoil the-

ory detailed in Ramesh et al. [39]. This theory is based on the time-stepping formulation given by Katz \& Plotkin [40], but eliminates the traditional small-angle assumptions in thin-airfoil theory which may be invalid in flows 


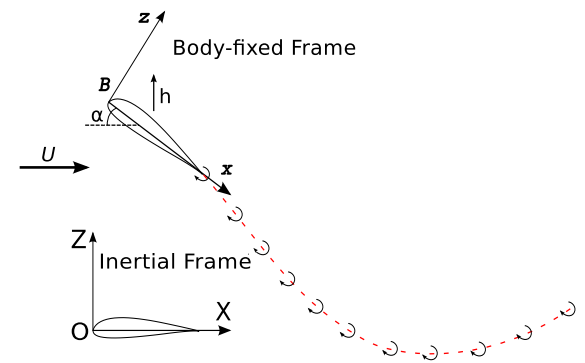

Figure 3: Depiction of time-stepping scheme.

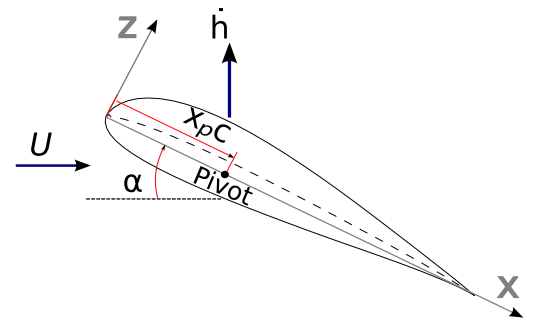

Figure 4: Airfoil velocities and pivot location.

of current interest. At each time step, a discrete vortex is shed from the airfoil trailing edge (referred to as TEV) as depicted in figure 3. When dictated by the LESP-based shedding criterion (section 2.2.2), a discrete vortex is also shed from the leading edge at some time steps. The vorticity distribution over the airfoil at any given time step is taken to be a Fourier series truncated to $r$ terms:

$$
\gamma(\theta)=2 U\left[A_{0} \frac{1+\cos \theta}{\sin \theta}+\sum_{i=1}^{r} A_{i} \sin (i \theta)\right]
$$

where the transformation variable $\theta$ relates to the chordwise coordinate as: $x=c(1-\cos \theta) / 2$, with $x$ measured from the leading edge; that is, $0 \leq x \leq c$ and $0 \leq \theta \leq \pi . A_{0}, A_{1}, \ldots, A_{r}$ are the time-dependent Fourier coefficients, and $U$ is the freestream velocity. The Kutta condition (zero vorticity at the trailing-edge) is enforced implicitly through the form of the Fourier series. The Fourier coefficients are calculated by enforcing the boundary condition of zero normal flow through the airfoil camberline as

$$
\begin{aligned}
& A_{0}=-\frac{1}{\pi} \int_{0}^{\pi} \frac{W(\theta)}{U} d \theta \\
& A_{i}=\frac{2}{\pi} \int_{0}^{\pi} \frac{W(\theta)}{U} \cos (i \theta) d \theta,
\end{aligned}
$$

where $W(\theta)$ is the induced velocity normal to the airfoil camberline. This value is calculated from components of motion kinematics, depicted in figure 4 , and induced velocities from all vortices in the flowfield. 
When there is no LEV shedding in a time step, the only unknown is the strength of the last-shed trailing-edge vortex and this is calculated iteratively such that Kelvin's circulation condition is satisfied [39]. If a TEV and LEV are both shed in a time step, their strengths are determined as discussed in section 2.2.2.

\subsection{2. $L E S P$ criterion for $L E V$ formation and shedding}

The LESP is a measure of the suction peak at the leading edge, which in turn is caused by the stagnation point moving away from the leading edge when the airfoil is at an angle of attack. From Garrick [41] and von Kármán $\&$ Burgers [42], the suction at the leading edge in potential flow may be expressed as

$$
S=\lim _{x \rightarrow L E} \frac{1}{2} \gamma(x) \sqrt{x}
$$

Evaluating using the current formulation, $S=\sqrt{c} U A_{0}$. The Leading Edge Suction Parameter is defined as a nondimensional value of suction at the leading edge, and is hence simply set equal to the first coefficient from Eq. (7), $A_{0}$.

As noted by Katz [43], real airfoils have rounded leading edges which can support some suction even when the stagnation point is away from the airfoil leading edge. The amount of suction that can be supported is a characteristic of the airfoil shape and Reynolds number of operation. When these quantities are constant, it was shown in Ramesh et al. [38] that initiation of LEV formation always occurred at the same value of LESP regardless of motion kinematics and history. This threshold value of LESP, which is a function of the airfoil shape and Reynolds number, is termed the critical LESP. This value, for any given airfoil and Reynolds number (and other specific operating conditions such as freestream turbulence and the presence of roughness), can be obtained from CFD or experimental predictions for a single motion [38], and can then be used for any other motion to predict LEV formation. In the LDVM model, a discrete vortex is shed is from the leading edge at those time steps when the instantaneous LESP ( $A_{0}$ value) is greater than the critical LESP value. The strength of the LEV is determined such that the the instantaneous LESP value, which would have otherwise exceeded the critical LESP value, is made equal to the latter. This condition, along with Kelvin's condition, is used to determine shed vortex strengths iteratively in time steps where both TEV and LEV are shed. 


\subsubsection{Vortex method details}

In the current approach, the vortex-core model proposed by Vatistas et al. [44], which gives an excellent approximation to the Lamb-Oseen vortex, is used to represent the discrete vortices as vortex blobs. Using this core model with order two, the velocities induced at $X$ and $Z(u$ and $w$ ) by the $k^{t h}$ vortex in the $X$ and $Z$ direction are:

$$
[u, w]=\frac{\gamma_{k}}{2 \pi} \frac{\left[\left(Z-Z_{k}\right),\left(X_{k}-X\right)\right]}{\sqrt{\left[\left(X-X_{k}\right)^{2}+\left(Z-Z_{k}\right)^{2}\right]^{2}+v_{\text {core }}^{4}}} .
$$

A nondimensional time step $\Delta t^{*}=\Delta t U / c=0.015$ is used for all simulations presented in this paper. Hald [45] has proved that the vortex-blob method is convergent (stable when run over long periods) so long as the vortex-core radius is larger than the average spacing between vortices. The average spacing between the vortices, $d$, is calculated as $d=c \Delta t^{*}$. The vortex core radius is taken to be approximately 1.3 times the average spacing between the vortices (as suggested by Leonard [46]): $v_{\text {core }}=0.02 c$. Convergence studies have been performed during the development of this method, and the numerical parameters have been selected such that the simulation results are not improved by either increasing or decreasing their values.

To control vortex count, and thus limit the computational cost, vortices which are a distance greater than ten chord lengths from the airfoil are deleted. When vortices are deleted from the domain, Kelvin's circulation condition which is used to iterate for shed vortex strengths is updated accordingly. Test simulations showed that results did not change when the cutoff distance was increased beyond ten chord lengths, implying that the velocity induced by vortices at a distance greater than ten chord lengths is negligible in comparison with other velocities acting on the airfoil.

At each time-step, all the free vortices in the flowfield are convected by the net local velocity induced at their centers. A first-order time-stepping procedure is used for updating vortex positions, since no significant change in accuracy was observed by using higher-order methods.

\subsubsection{Forces and moment on airfoil}

The forces and moment on the airfoil are derived in detail in Ramesh et al. [38] and are outlined briefly here. The two forces on the airfoil are the normal force and leading-edge suction force, given by 


$$
\begin{aligned}
F_{N}= & \rho \pi c U\left[(U \cos \alpha+\dot{h} \sin \alpha)\left(A_{0}+\frac{1}{2} A_{1}\right)+c\left(\frac{3}{4} \dot{A}_{0}+\frac{1}{4} \dot{A}_{1}+\frac{1}{8} \dot{A}_{2}\right)\right] \\
& +\rho \int_{0}^{c}\left(\left(\frac{\partial \phi_{l e v}}{\partial x}\right)+\left(\frac{\partial \phi_{\text {tev }}}{\partial x}\right)\right) \gamma(x) d x
\end{aligned}
$$

$$
F_{S}=\rho \pi c U^{2}\left(A_{0}\right)^{2}
$$

The moment about an arbitrary reference point, $x_{r e f}$, is given by

$$
\begin{aligned}
M= & x_{r e f} F_{N}-\rho \pi c^{2} U\left[(U \cos \alpha+\dot{h} \sin \alpha)\left(\frac{1}{4} A_{0}+\frac{1}{4} A_{1}-\frac{1}{8} A_{2}\right)\right. \\
& \left.+c\left(\frac{7}{16} \dot{A}_{0}+\frac{11}{64} \dot{A}_{1}+\frac{1}{16} \dot{A}_{2}-\frac{1}{64} \dot{A}_{3}\right)\right] \\
& -\rho \int_{0}^{c}\left(\left(\frac{\partial \phi_{\text {lev }}}{\partial x}\right)+\left(\frac{\partial \phi_{\text {lev }}}{\partial x}\right)\right) \gamma(x) x d x .
\end{aligned}
$$

The force coefficients $\left(C_{N}\right.$ and $\left.C_{S}\right)$ are evaluated by dividing the forces by $\frac{1}{2} \rho U^{2} c$ and the moment coefficient $\left(C_{m}\right)$ is obtained as $M / \frac{1}{2} \rho U^{2} c^{2}$. The

lift and drag coefficients $\left(C_{l}\right.$ and $\left.C_{d}\right)$ are calculated using components of normal and leading-edge suction forces. The time derivatives of the Fourier coefficients in the forces and pitching moment (equations 12-14) arise from the apparent-mass contribution which is very significant for high-reducedfrequency motion.

\subsubsection{Limitations of the aerodynamics model}

As shown in [38], the predictions from the current LDVM are in reasonable, and sometimes excellent, agreement with those from CFD and experiments. Because the LDVM does not model thick or separated boundary layers, the method is restricted to motions where the LEV formation occurs without being accompanied by significant trailing-edge separation or stall. For most rounded-leading-edge airfoils, the LDVM is most reliable for highreduced-frequency motions, with $k>0.4$. In the current work, all LCOs studied in section 4 have $k>0.6$.

Another disadvantage, which is characteristic of vortex methods, is the exponential increase in computational time with number of vortices in the 
flow field $\left(\mathcal{O}\left(n^{2}\right)\right)$. Fast summation methods [47], amalgamation of vortices, or deletion of vortices that exit the field of interest could be used to control the vortex count. As mentioned previously, vortices that are at a distance greater than ten chord lengths from the airfoil are deleted in the current implementation.

\subsection{Aero-structural coupling}

The coupling between the structural and aerodynamic models is described next. The structural model is governed by second-order ordinary differential equations in continuous time, whereas the aerodynamic model is naturally written in discrete time. In order to couple both models, the structural equations are integrated in time using a three-step Adam-Bashforth scheme [48], whereby the generalized coordinates and their derivatives (pitch, plunge and corresponding rates) are marched as

$$
\left\{\begin{array}{c}
\boldsymbol{q} \\
\dot{\boldsymbol{q}}
\end{array}\right\}^{n+1}=\left\{\begin{array}{c}
\boldsymbol{q} \\
\dot{\boldsymbol{q}}
\end{array}\right\}^{n}+\frac{\Delta t}{12}\left[23\left\{\begin{array}{c}
\dot{\boldsymbol{q}} \\
\ddot{\boldsymbol{q}}
\end{array}\right\}^{n}-16\left\{\begin{array}{c}
\dot{\boldsymbol{q}} \\
\ddot{\boldsymbol{q}}
\end{array}\right\}^{n-1}+5\left\{\begin{array}{c}
\dot{\boldsymbol{q}} \\
\ddot{\boldsymbol{q}}
\end{array}\right\}^{n-2}\right]
$$

with $n$ being the time step at which states are evaluated. This is an explicit time-marching scheme in which only information from previous time steps is required.

The aero-structural integration is based on a loosely-coupled approach in which information is exchanged at each time step, but no subiterations are included. This scheme has been chosen due to the first-order, explicit nature of the aerodynamic model, and because it has shown excellent convergence properties for the appropriate selection of simulation parameters. The main steps of the process are briefly summarized below:

1. Based on the geometry and velocities at time step $n$, the aerodynamic loads are computed from the discrete-vortex method with the corresponding vorticity distribution, including leading-edge vortices when applicable.

2. These aerodynamic loads at time step $n$ are applied to the structural equations, Eq. (6), which are solved to yield the acceleration values $\ddot{h}^{n}$ and $\ddot{\alpha}^{n}$.

3. From the Adam-Bashforth integration scheme, Eq. (15), the structural states (plunge, pitch and corresponding rates) at time step $n+1$ are determined. 
4. The procedure is repeated from step 1.

The aeroelastic coupling and integrations are also depicted in a flowchart in Figure 5, with a detailed description of how the discrete-vortex method operates.

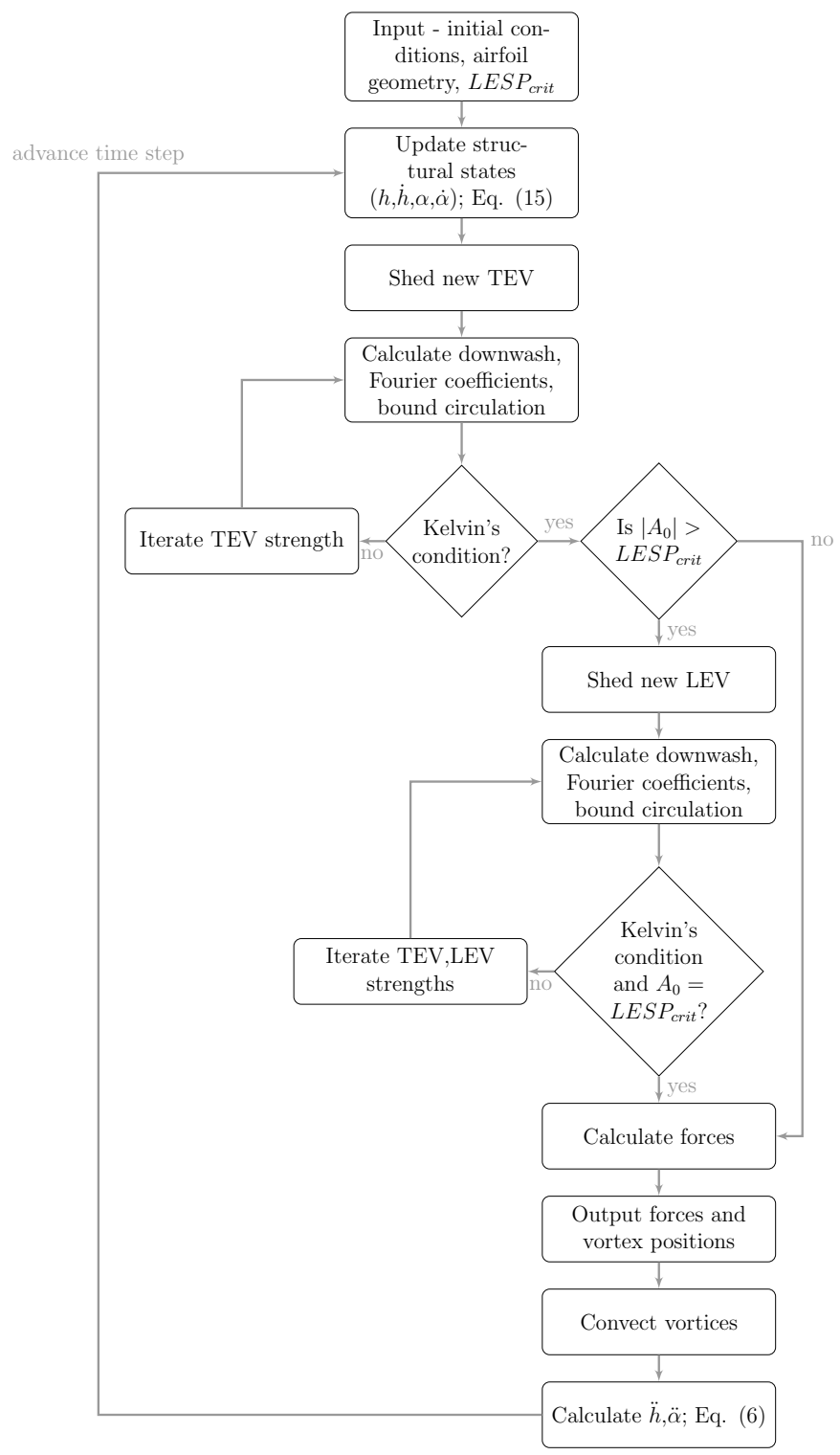

Figure 5: Flowchart summarizing aero-structural integration. 


\section{Validation against previous work}

Validation of the current method with linear aerodynamics is presented for aeroelastic predictions: using linear structures in section 3.1 and using nonlinear structures in section 3.2. Because there is almost no suitable data in the literature for passive airfoil aeroelasticity in high-frequency, LEVdominated flows, validation of the nonlinear aerodynamics is presented for prescribed kinematics in section 3.3.

\subsection{Validation for linear aerodynamics and linear structures: Onset of linear flutter for the classical 2-DOF airfoil}

The classical two-degree-of-freedom linear flutter problem is used to validate the aeroelastic model developed in this work. The LEV shedding in the aerodynamic model is "turned off" by setting the critical LESP to a very high value of 5.0. The aerodynamic model thus provides a potential-flow solution with attached flow at the leading edge, enabling validation of the method with linear-flutter-onset data. Since the current method is based in the time domain, flutter velocities are identified as those above which divergent oscillations occur.

As an illustration of how the oscillation characteristics vary with velocity, figure 6 shows the variations of $\alpha$ with $t^{*}$ at three nondimensional velocity values $\left(U^{*}=U / \omega_{\alpha} c\right)$ for an example configuration. It is seen that the oscillatory behavior changes from convergent for the smallest velocity value, to constant amplitude at the intermediate value, to divergent at the highest value. Thus, it is possible to determine the nondimensional flutter velocity $\left(U_{F}^{*}=U_{F} / \omega_{\alpha} c\right)$ using the current approach as that value at which the oscillation amplitude remains invariant with time.
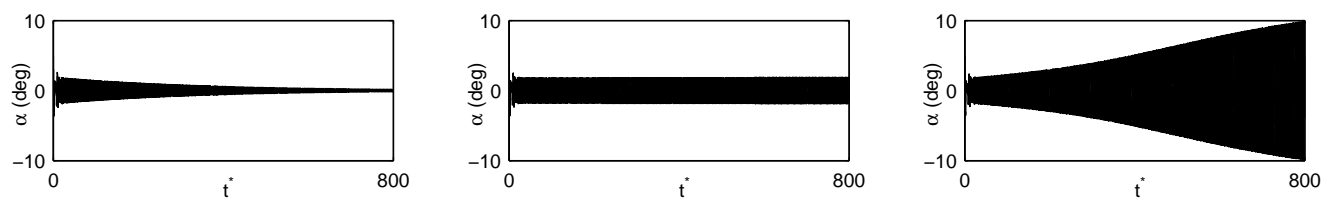

Figure 6: Variation of oscillatory behavior with velocity for an example configuration $\left(x_{p}=0.35, \kappa=0.05, r_{\alpha}=0.5, x_{\alpha}=0.2\right.$ and $\left.\bar{\omega}=1.0\right)$. Left to right: $U^{*}=0.62$, $U^{*}=0.64$, and $U^{*}=0.66$. Constant amplitude for the center case corresponds to the linear flutter velocity of $U_{F}^{*}=0.64$ for this example. 


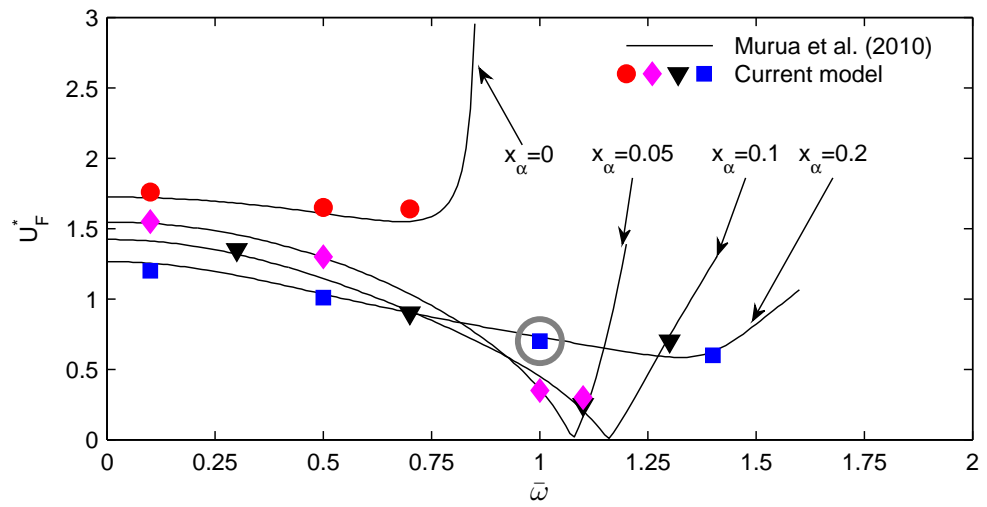

Figure 7: Flutter-onset velocities from current method compared against data published by Murua et al. [49]. Structural parameters: $x_{p}=0.35, \kappa=0.05, r_{\alpha}=0.5$. The gray circle contains the data point which is determined through the illustration in figure 6 .

In figure 7 , flutter-onset velocities for a range of frequency ratios $(\bar{\omega}=$ $\left.\omega_{h} / \omega_{\alpha}\right)$ and static unbalance values $\left(x_{\alpha}\right)$ are compared against data published by Murua et al. [49]. It is seen that the comparison between the two methods is excellent, thus validating the current approach for cases with linear aerodynamics and linear structures. Small deviations may be attributed to departures in the current aerodynamic model from Theodorsen's theory: modeling of wake roll-up in the current model instead of a flat wake used in classical theory and removal of the small-angle approximations typically made in classical theory.

\subsection{Validation for linear aerodynamics and nonlinear structures: Onset of flutter for soft springs and LCO amplitudes for hard springs}

Structural nonlinearities due to nonlinear stiffness can be modeled in the current work as described in equations 5. Aeroelastic behaviors resulting from the coupling of such structural nonlinearity, by use of different cubic stiffnesses, with linear aerodynamic models are documented in Lee et al. [17]. Specifically, two types of cubic nonlinearities were considered for the pitch spring: a soft spring for which $\beta_{\alpha}$ is negative and a hard spring for which $\beta_{\alpha}$ is positive. Results from that paper are used to validate the current aerostructural model. As was done in the earlier subsection, LEV shedding is "turned off" to simulate linear aerodynamics resulting from attached leadingedge flows. 

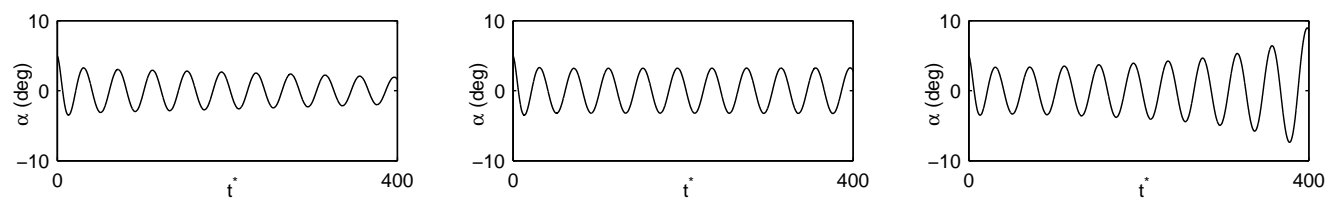

Figure 8: Variation of oscillatory behavior with velocity for an example configuration having a soft pitch spring with initial pitch amplitude of $\alpha(0)=5^{\circ}$. Structural parameters are $x_{p}=0.25, \kappa=0.01, r_{\alpha}=0.5, x_{\alpha}=0.25, \bar{\omega}=0.2, \beta_{\alpha}=-3, U_{F}^{*}=3.15$. Left to right: $U^{*} / U_{F}^{*}=0.9905, U^{*} / U_{F}^{*}=0.9920$, and $U^{*} / U_{F}^{*}=0.9936$. For $\alpha(0)=5^{\circ}$, it is seen that the response is divergent for $U^{*}>0.9920 U_{F}^{*}$.

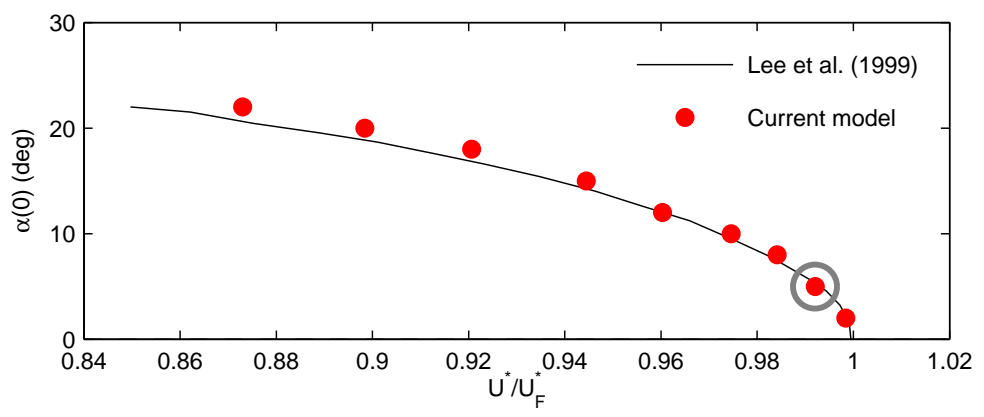

Figure 9: Flutter-onset conditions for a soft pitch spring from current method compared against data published by Lee et al. [17]. Structural parameters: $x_{p}=0.25, \kappa=0.01$, $r_{\alpha}=0.5, x_{\alpha}=0.25, \bar{\omega}=0.2, \beta_{\alpha}=-3$. The gray circle contains the data point which is determined through the illustration in figure 8 .

As shown in Lee et al. [17], when a soft pitch spring is used, divergent oscillations can occur even at velocities below the linear flutter velocity, $U_{F}$, provided a sufficiently large initial pitch amplitude is used. Figure 8 shows the oscillatory behavior for an initial pitch angle of $\alpha(0)=5^{\circ}$ predicted by the current method for an example configuration with a soft pitch spring for three values of $U^{*} / U_{F}^{*}$ below unity. It can be seen that the oscillatory behavior changes from convergent to constant-amplitude to divergent. Figure 9 compares the prediction of $U^{*} / U_{F}^{*}$ for flutter onset as a function of the initial pitch angle, $\alpha(0)$, for the same soft-spring configuration with results published in Lee et al. [17]. The comparison is seen to be excellent.

Lee et al. [17] also show that, with a hard spring, the oscillations are always convergent for velocities that are less than the linear flutter speed, i.e. for $U^{*} / U_{F}^{*}<1.0$. For $U^{*} / U_{F}^{*}>1.0$, the nonlinear spring stiffness results in the formation of LCOs rather than the divergent oscillations that would 
have occurred with a linear spring. The amplitude of the resulting LCO, $\alpha_{A}$, is independent of the initial conditions in pitch and plunge, but increases with increasing $U^{*} / U_{F}^{*}$. The behavior is illustrated for an example configuration with a hard pitch spring for three values of $U^{*} / U_{F}^{*}$ in figure 10. Comparison of the predicted variation of LCO amplitude with $U^{*} / U_{F}^{*}$ from the current method with the results of Lee et al. [17], shown in figure 11, is seen to be excellent. The small deviations from the results of Lee et al. [17] (which uses linear aerodynamics), which are noticeable in the larger-amplitude oscillations, may be attributed to the removal of small-angle approximations and the use of wake rollup in the current aerodynamic model.
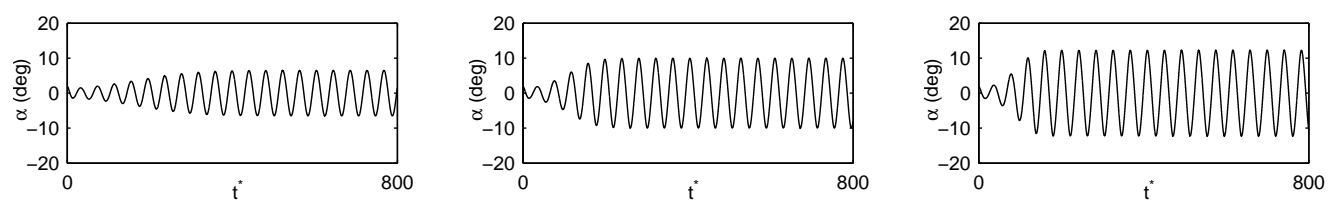

Figure 10: Behavior of LCO for three values of $U^{*} / U_{F}^{*}$ for an example configuration having a hard pitch spring. Structural parameters are $x_{p}=0.25, \kappa=0.01, r_{\alpha}=0.5, x_{\alpha}=0.25$, $\bar{\omega}=0.2, \beta_{\alpha}=3, U_{F}^{*}=3.15$. Left to right: $U^{*} / U_{F}^{*}=1.006, U^{*} / U_{F}^{*}=1.029$, and $U^{*} / U_{F}^{*}=1.048$.

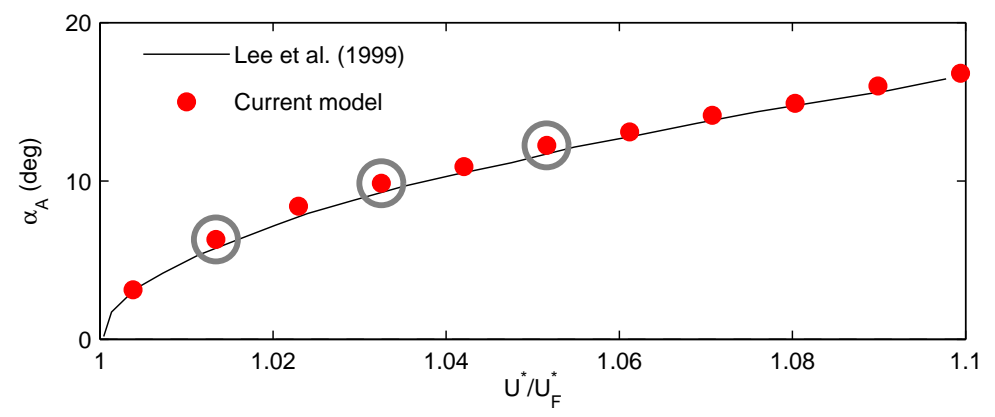

Figure 11: Variation of LCO amplitude with $U^{*} / U_{F}^{*}$ for a hard pitch spring from current method compared against data published by Lee et al. [17]. Structural parameters: $x_{p}=$ $0.25, \kappa=0.01, r_{\alpha}=0.5, x_{\alpha}=0.25, \bar{\omega}=0.2, \beta_{\alpha}=3$. The gray circles contain the data points which are determined through the illustration in figure 10. 


\subsection{Validation of nonlinear aerodynamic model for prescribed kinematics:} Force coefficients for vortex-dominated flows, typical of power-extraction regimes

The LDVM aerodynamic method has been validated against CFD and experiment in Ramesh et al. [38] for several airfoils undergoing prescribed unsteady motions at low Reynolds numbers. In this paper, one of these validation cases from [38] is adapted and briefly presented for completeness. In this example, a prescribed sinusoidal pitch-plunge motion of a NACA 0015 airfoil at a Reynolds number of 1,100 and high reduced frequency (suitable for this model) of $k=0.439$ is used to validate the LDVM aerodynamic model for a case with intermittent LEV shedding. Force coefficients and flowfield predictions from the current aerodynamic model are compared against those presented by Kinsey \& Dumas [26], who used laminar runs of the FLUENT CFD code [50] to study oscillating motions for power extraction from a moving fluid. As discussed in greater detail in Ramesh et al. [38], the critical-LESP value for this case (NACA 0015 airfoil at a Reynolds number of 1,100$)$ was obtained from CFD to be 0.19 . The pitch-plunge kinematics are defined as

$$
\begin{aligned}
\frac{h}{c}(t) & =\cos (0.28 \pi t), \\
\alpha^{o}(t) & =76.33^{\circ} \cos \left(0.28 \pi t+\frac{\pi}{2}\right) .
\end{aligned}
$$

Figure 12 compares steady-state force and pitching-moment predictions from LDVM (at the 5th cycle) with the CFD results of Ref. [26] for one period $(T)$ of the prescribed motion. In all four subplots, the pitch angle variation, $\alpha(t / T)$ is plotted in gray with the scale shown on the right side. Figure 12(a) shows the time variation of LESP for the motion, with the positive and negative values of the critical LESP of 0.19 marked as dashed lines. It is seen that the LESP starts to decrease at the start of the cycle, reaching and staying at the negative critical value for approximately a quarter of the cycle. With increasing pitch angle, the LESP increases and reaches the positive critical value in the second half of the cycle, staying at that value again for approximately a quarter of the cycle. During the time when the LESP is at the positive/negative critical value, clockwise/counter-clockwise discrete vortices are generated, which form an LEV that is shed from the upper/lower surface. Figures 12(b)-(d) compare the variations of lift, drag, and moment 


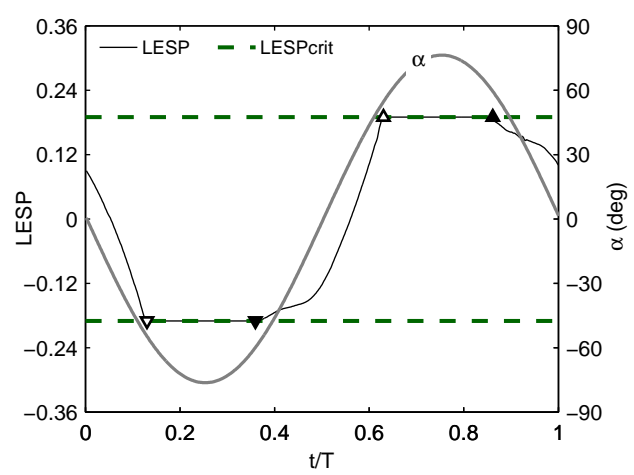

(a)

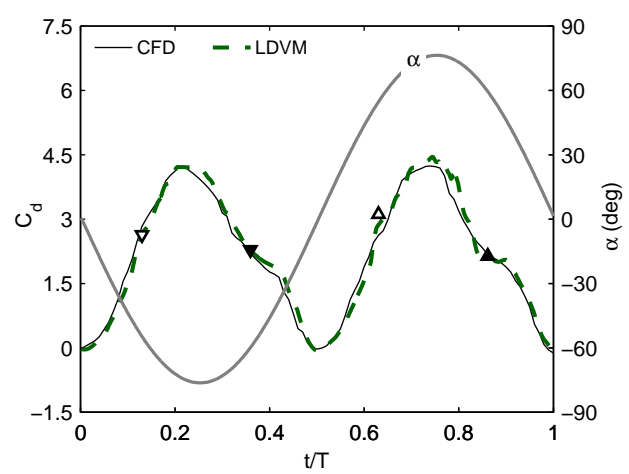

(c)

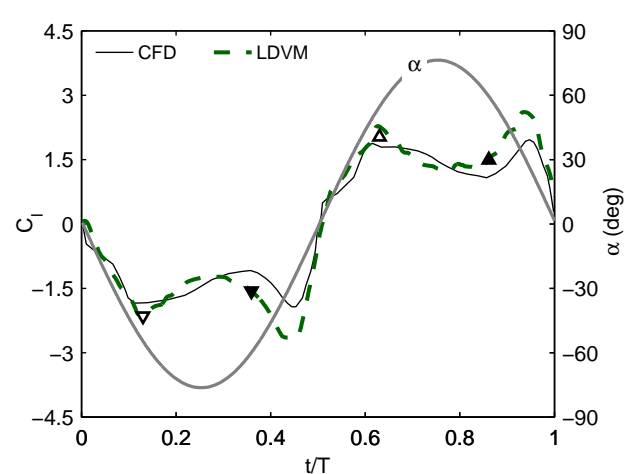

(b)

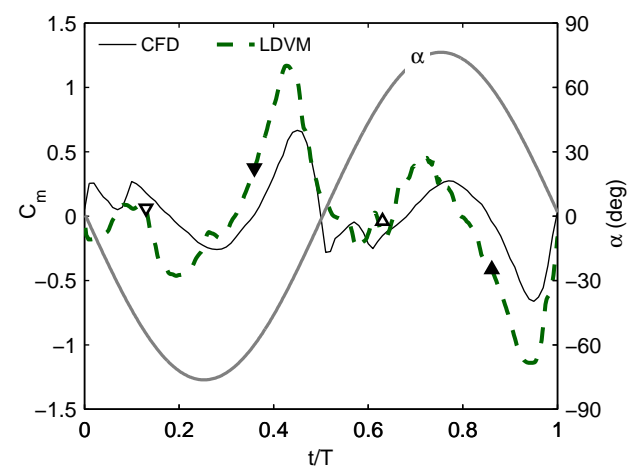

(d)

Figure 12: Variation with $t / T$ of: (a) LESP from current model, (b) lift coefficient from LDVM and CFD, (c) drag coefficient from LDVM and CFD, (d) pitching moment coefficient about one-third chord from LDVM and CFD. The CFD solutions are from Ref. [26]. In all plots, the initiation and termination of LEV shedding is marked on the LDVM curves using open and filled triangle symbols respectively, with upward/downward-pointing triangles indicating upper/lower-surface LEV shedding.

coefficients from the LDVM and CFD predictions. The comparison in lift is seen to be very good, with the low-order method capturing all of the trends that are seen in the CFD prediction. The comparison in drag is excellent. Pitching-moment prediction from the low-order method is seen to have some discrepancies compared to the CFD result, but the general behavior is similar between the two methods. The discrepancies in pitching-moment prediction are attributed to the formation of thick or separated boundary layers on the airfoil surfaces, not modeled in the LDVM theory [38].

The flow prediction from LDVM is compared with the CFD vorticity plot provided in Ref. [26] for $t / T=1$ (at which $h / c=1$ and $\alpha=0$ ) in figure 13 . 


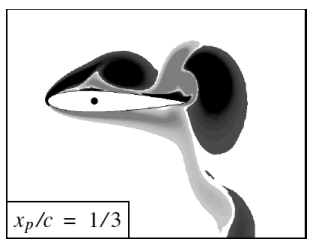

CFD

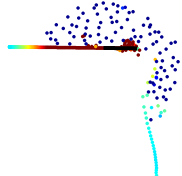

LDVM

Figure 13: Vorticity plot from CFD (Kinsey \& Dumas [26], reproduced with permission) for $t / T=1.0$ compared with flowfield from LDVM for $t / T=1.0$.

Concentrations of vorticity are seen at the same locations with respect to the airfoil in both predictions.

This validation, along with others presented in [38], demonstrate that the LDVM approach is ideally suited for use as the aerodynamic model in the current work. It is successful in predicting the aerodynamic loads to sufficient accuracy even in the presence of intermittent LEV shedding. Of importance to the current work is that the LDVM is also computationally fast, with runs times being typically less than 1 minute for 1 cycle compared to several hours for a high-order CFD method.

\section{Study of high-frequency LCOs in low-Reynolds-number flows}

The characteristics of limit-cycle oscillations in low-Reynolds-number, high-reduced-frequency flows are studied using the numerical model described in section 2. From equation 6, the structural parameters which influence aeroelastic behavior are static unbalance $\left(x_{\alpha}\right)$, radius of gyration $\left(r_{\alpha}\right)$, inverse mass ratio $(\kappa)$, frequency ratio $\left(\bar{\omega}=\omega_{h} / \omega_{\alpha}\right)$ and the cubic stiffening constants $\left(\beta_{h}\right.$ and $\left.\beta_{\alpha}\right)$. The aerodynamic parameters are the freestream velocity $(U)$ and the critical LESP used in the discrete-vortex method; the critical-LESP value is a function of airfoil shape and Reynolds number.

\subsection{Selection of parameter space}

The parameter space in this study is confined to combinations of structural and aerodynamic parameters which result in high-frequency LCOs. Typical values from the literature are used for the radius of gyration, $r_{\alpha}=$ 0.5 ; inverse-mass ratio, $\kappa=0.05$; and pivot, $x_{p}=0.35$. To determine the range of static unbalance $\left(x_{\alpha}\right)$ and frequency ratio $(\bar{\omega})$ that result in highfrequency LCOs, linear flutter velocity data and the values of oscillation frequencies at these speeds from Murua et al. [49] are used. Reduced frequency, 
$k=\omega c /(2 U)$, of the neutrally-stable response at flutter velocity is calculated from this data over a range of $x_{\alpha}$ and $\bar{\omega}$, and is plotted in figure 14. It is seen that frequency ratios between 1 and 1.3, and static unbalance values of 0.05 , 0.1 and 0.2 , result in high reduced frequencies well above the desired lower limit of $k=0.6$ as discussed in section 2.2.5 and shown by the gray line in figure 14. Hence this space in $x_{\alpha}$ and $\bar{\omega}$ is chosen for this study. Although the reduced frequencies for LCOs above the flutter velocity would be different from the oscillation frequencies at $U_{F}$, the latter provide a useful guide to identify the parameter space of interest. It is noted that these values of $x_{\alpha}$ and $\bar{\omega}$ would also be of interest for power extraction because of the low flutter speeds (cut-in speeds). The Reynolds number for the study is chosen to be 1,000. The $L E S P_{\text {crit }}$ for a fixed Reynolds number depends on the airfoil's leading-edge radius, and may vary from zero (perfectly sharp leading edge) to an upper limit of 0.3 . Higher values of $L E S P_{c r i t}$ are unrealistic to consider since trailing-edge separation/"bluff body"-type flow would result if the airfoil's leading edge were excessively rounded.

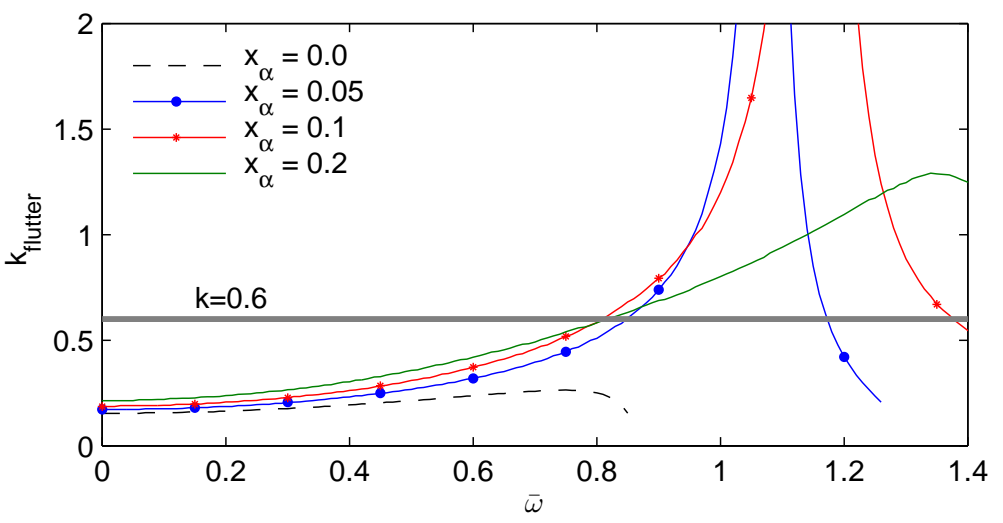

Figure 14: Reduced-frequency variation with $x_{\alpha}$ and $\bar{\omega}$ at the flutter velocity, derived from data published by Murua et al. [49]. Structural parameters: $x_{p}=0.35, \kappa=0.05, r_{\alpha}=0.5$.

The parameter space outlined above is employed in the remainder of section 4 . In section 4.2 , a baseline case is defined and the LCO properties for this case are studied in detail. In sections 4.3- 4.6, the variations in LCO properties with deviations in parameters from the baseline case are analyzed.

\subsection{Baseline case for the four-part parametric study}

The base parameter set for this research is derived from the considerations detailed above and is listed in table 1 . Values of $x_{\alpha}=0.05$ and $\bar{\omega}=1.0$ 


\begin{tabular}{|c|c|c|}
\hline Parameter & Symbol & $\underline{\text { Value }}$ \\
\hline Static unbalance & $\overline{x_{\alpha}}$ & 0.05 \\
\hline Radius of gyration & $r_{\alpha}$ & 0.5 \\
\hline Inverse mass ratio & $\kappa$ & 0.05 \\
\hline Frequency ratio & $\bar{\omega}=\omega_{h} / \omega_{\alpha}$ & 1.0 \\
\hline Cubic stiffening-pitch & $\beta_{\alpha}$ & 0.0 \\
\hline Cubic stiffening-plunge & $\beta_{h}$ & 0.0 \\
\hline Flutter velocity & $U_{F}^{*}$ & 0.359 \\
\hline Freestream velocity & $U^{*}$ & $1.3 U_{F}^{*}=0.4667$ \\
\hline Critical LESP & $L E S P_{\text {crit }}$ & 0.11 \\
\hline Initial conditions-pitch & $\alpha(0), \dot{\alpha}(0)$ & $\alpha(0)=10^{\circ}, \dot{\alpha}(0)=0$ \\
\hline Initial conditions-plunge & $h(0), \dot{h}(0)$ & $h(0)=\dot{h}(0)=0$ \\
\hline
\end{tabular}

Table 1: Base parameter set used to study LCO characteristics in high-frequency, lowReynolds-number flows.

are used, for which the linear flutter velocity from Ref. [49] is $U_{F}^{*}=0.359$. The baseline freestream velocity is taken to be 1.3 times the linear flutter velocity. A $2.3 \%$-thick flat-plate airfoil with a semi-circular leading edge is considered, and the critical LESP value for this airfoil at $R e=1,000$ is 0.11 as calibrated from CFD in Ramesh et al. [38]. The springs are assumed to have linear stiffness in the baseline configuration. The effect of cubic stiffening is analyzed in section 4.4 .

The airfoil's aeroelastic response for the base parameter set listed in table 1 is shown in figure 15 . The pitch and plunge amplitudes increase from their initial values and reach a limiting value as shown in the insets of figures 15(a) and 15(b). The variations of pitch and plunge with time (nondimensional), after limit-cycle oscillations are reached, are plotted in figures 15(a) and (b). It is apparent and is further established below, that the response is single-period. A single time period of the airfoil's response is enclosed by dashed lines. Figure 15(c) shows the variation of LESP with $t^{*}$. This parameter controls leading-edge vortex formation in the discrete-vortex model. From the figure, it is seen that during one period, the LESP value reaches the positive and negative critical LESP values once in each cycle. This behavior corresponds to one LEV being formed on the airfoil's upper surface followed by another on the lower surface in one period. The time instants at which the LESP values overlap with the critical LESP value in the figure, mark the instants at which discrete vortices are released from the leading edge in the discrete-vortex model. The lift, drag and pitching moment coefficients calculated from the aerodynamic model are shown in 

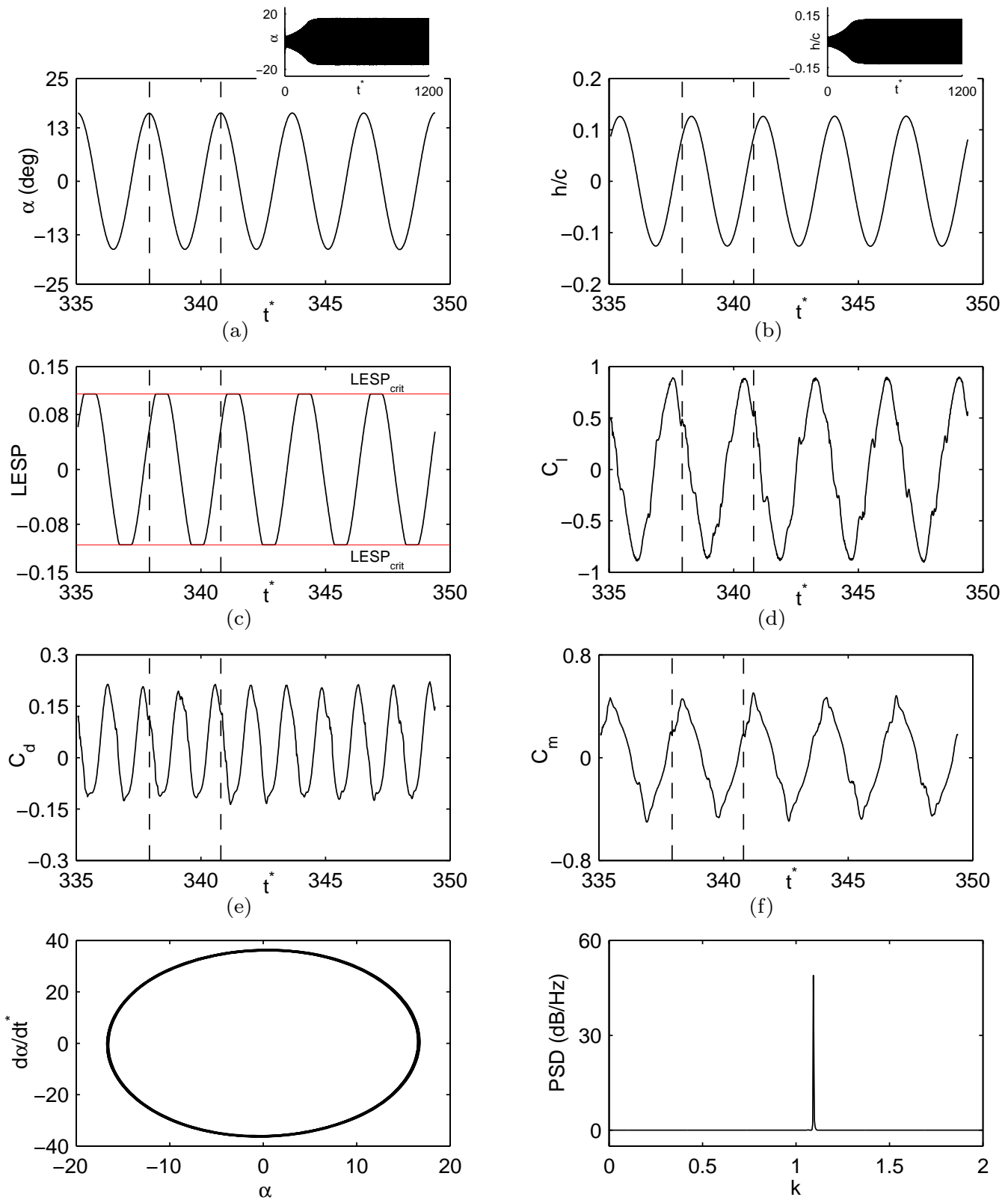

(g)

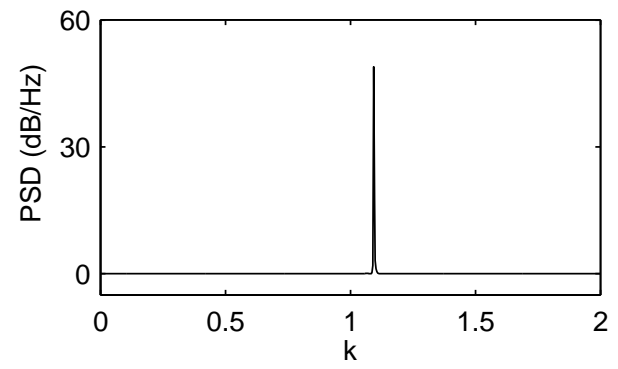

(h)

Figure 15: Baseline case: limit-cycle response for the parameters listed in table 1. Time variation of (a) pitch angle, (b) plunge per unit chord, (c) LESP, (d) lift coefficient, (e) drag coefficient, (f) pitching moment coefficient. The dashed lines enclose one period of the LCO. (g) and (h) are phase-plane and PSD plots respectively. Insets in (a) and (b) show long-time responses for pitch angle and plunge per unit chord. 
figures 15(d)-(f). Figures 15(g) and (h) are phase-plane and power spectral density (PSD) plots of the response, respectively. The horizontal axis of the PSD plot is reduced frequency $(k)$. The phase-plane and PSD plots further affirm that the limit-cycle oscillation is single period. The PSD plot shows the response reduced frequency to be approximately 1.08. Thus the airfoil oscillation ensuing from the chosen parameters is of high reduced frequency, where the flow is expected to be dominated by leading-edge vortices and apparent-mass forces, and the aerodynamic model is expected to represent the flow physics well.

The steady-state and harmonic limit-cycle oscillations in pitch and plunge may be represented as,

$$
\begin{aligned}
\alpha & =\alpha_{A} \cos \left(2 k t^{*}\right), \\
\frac{h}{c} & =\frac{h_{A}}{c} \cos \left(2 k t^{*}+\phi\right),
\end{aligned}
$$

where $\alpha_{A}$ and $h_{A}$ are the amplitudes of pitch and plunge, $k$ is the reduced frequency of oscillation, and $\phi$ is the phase angle between pitch and plunge (with pitch leading plunge). For the LCO illustrated in figure 15, $\alpha_{A}=16.6^{\circ}$, $h_{A}=0.128 c, k=1.08$ and $\phi=49.1^{\circ}$.

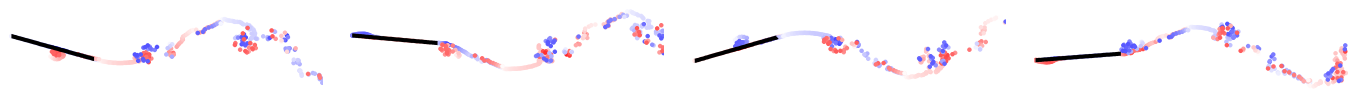
(a) $t^{*} / T^{*}=0$
(b) $t^{*} / T^{*}=0.25$
(c) $t^{*} / T^{*}=0.5$
(d) $t^{*} / T^{*}=0.75$

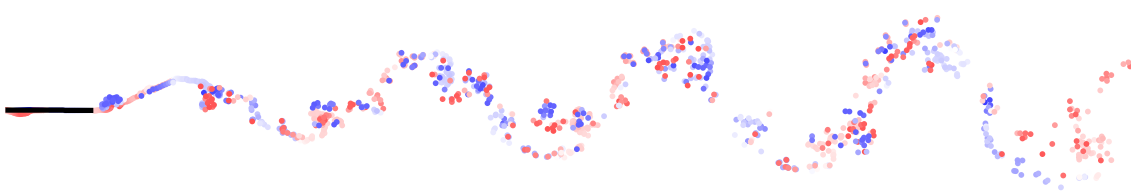

(e) Wake structure.

Figure 16: Baseline case: flow topology plots for the limit-cycle response obtained using the parameters listed in table 1. Plots (a)-(d) depict vorticity distributions at four equally spaced time intervals over one period of the oscillation. Plot (e) shows the wake structure resulting from the LCO.

Figures 16(a)-(d) depict the flow topology during one period of the LCO. As noted earlier, one LEV is shed over the airfoil upper surface, followed by another from the lower surface during one cycle. In (b) and (d) discretevortex shedding from the leading edge is observed on the upper and lower 
surfaces respectively. In (a) and (c) the LEVs are seen convecting over the airfoil chord, on the lower and upper surfaces, respectively. Figure 16(e) depicts the wake topology ensuing from the limit-cycle oscillation.

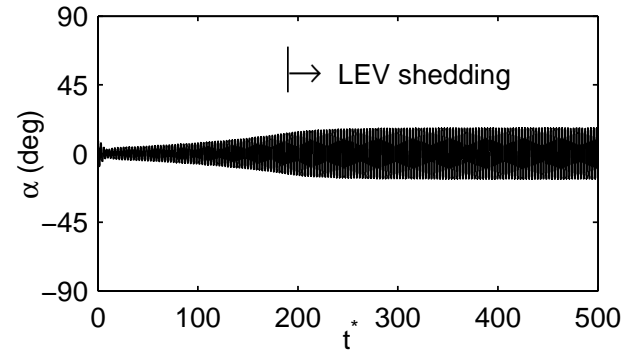

(a)

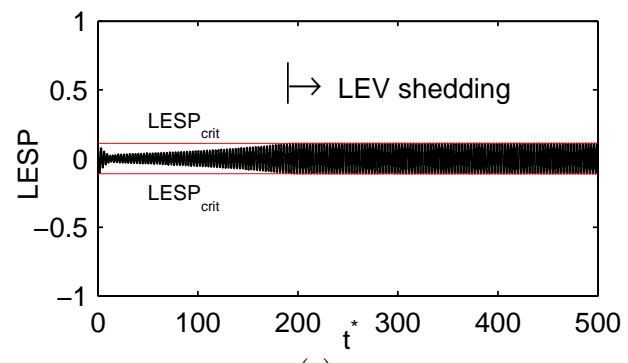

(c)

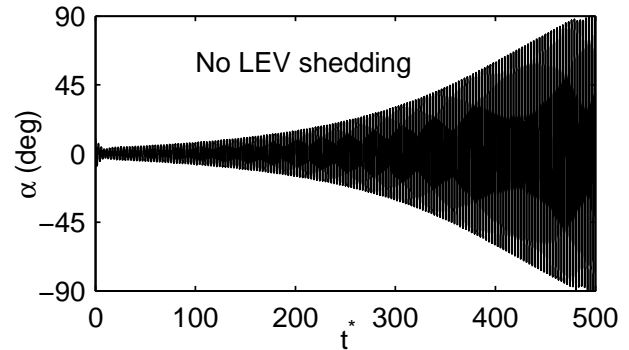

(b)

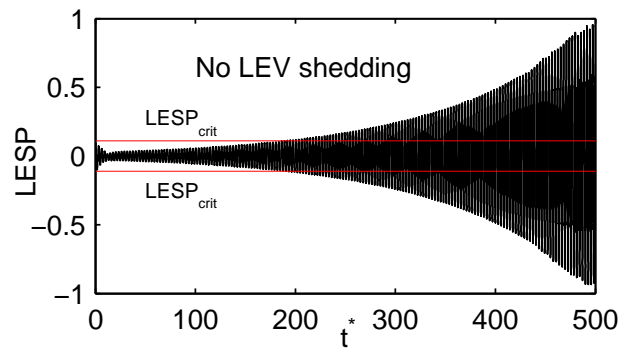

(d)

Figure 17: Illustration of aerodynamic nonlinearity, caused by LEV shedding, as the reason for LCO formation instead of divergent oscillations in the baseline case. (a) Pitch response with LEV shedding, (b) pitch response without LEV shedding, (c) LESP variation with LEV shedding, (d) LESP variation without LEV shedding.

\subsection{Parametric study A: Effect of change in freestream velocity}

The effect of freestream velocity on LCO characteristics is first illustrated by considering three representative values of $U^{*} / U_{F}^{*}$. Subsequently, the LCO behavior is presented for a wide range of $U^{*} / U_{F}^{*}$ to show the resulting bifurcation characteristics. It is recalled that, in this study, $U_{F}^{*}=0.359$ is the linear flutter velocity from Ref. [49].

The LCO behaviors for the three velocities are presented in figure 18 by plotting in three columns the oscillations at three values of $U^{*} / U_{F}^{*}$ of 1.3 , 1.8, and 2.3, from left to right. The top row shows the oscillations of $\alpha$ for a representative time window of $335 \leq t^{*} \leq 385$. Also marked, using circle symbols, on the right side of each subplot of the top row are the $\alpha$ 

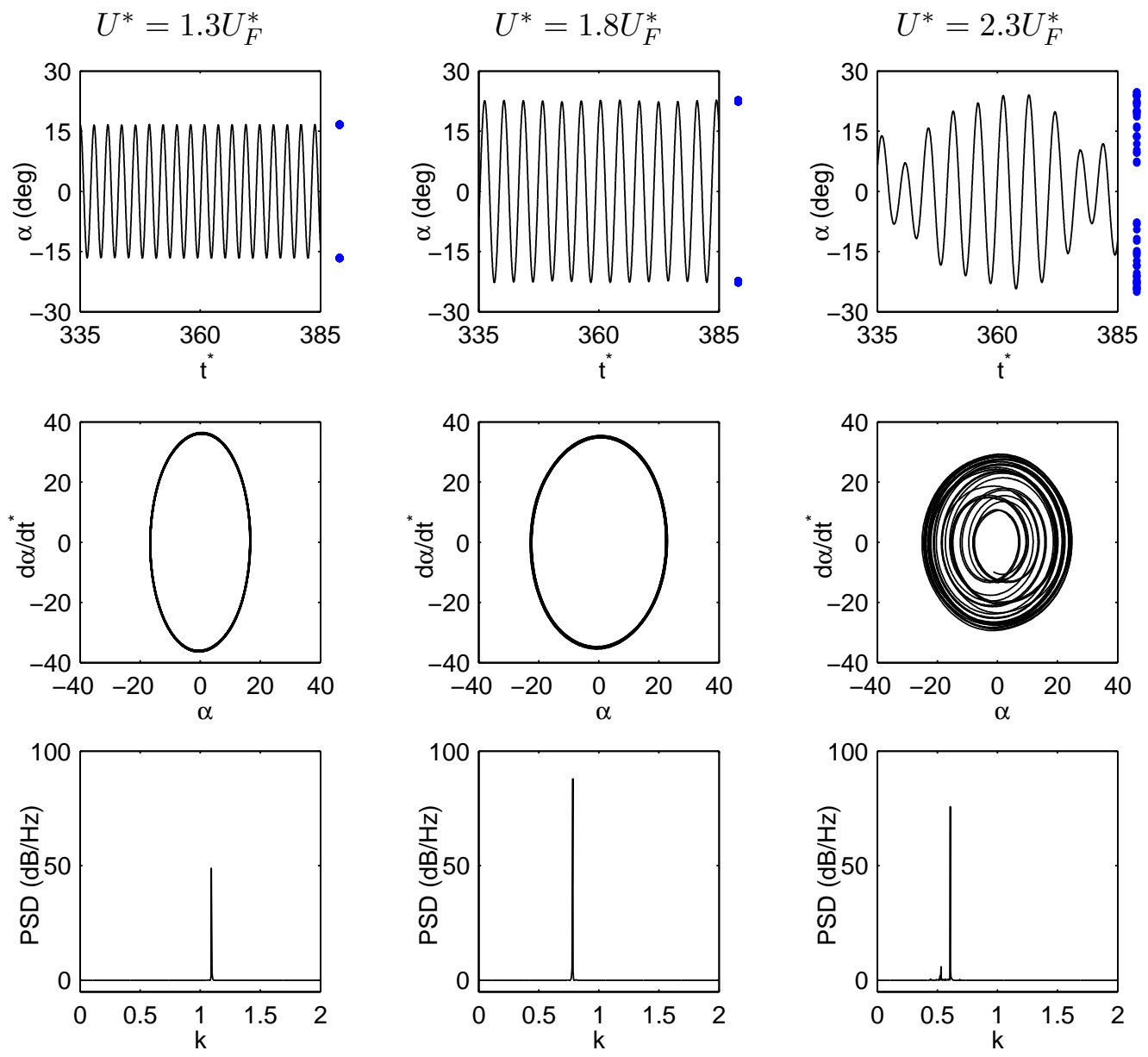

Figure 18: Parametric study A: comparison of LCO characteristics for different values of freestream velocity. Top row: time variation of pitch angle; Middle row: phase-plane plots; Bottom row: power-spectral density plots. Circle symbols on the right side of each top-row subplot show the pitch values corresponding to all the positive and negative peaks in pitch angle-vs-time variation.

values corresponding to all the positive and negative peak values in the $\alpha-t^{*}$ variation. These peak values are identified by determining the time instants at which $d \alpha / d t^{*}=0$. For the first two $U^{*} / U_{F}^{*}$ values of 1.3 and 1.8 , it is seen that the $\alpha-t^{*}$ variations are single-period oscillations. For each of these two cases, all points for the positive peaks have the same value of $\alpha$, resulting in all the symbols on the right side of the plot coinciding with each other. The same behavior is true for the negative peaks as well. Going from $U^{*} / U_{F}^{*}$ of 
1.3 to 1.8 , it is seen that the amplitude of $\alpha$ oscillations increases, while the frequency decreases. The behavior for the highest velocity, $U^{*} / U_{F}^{*}=2.3$, is seen to be different from those for the first two velocities. The oscillations for this case are seen to have multiple amplitudes. As a result, the symbols for the $\alpha$ values for the positive and negative peaks on the right side of the subplot do not coincide, but have a spread over an $\alpha$ range.

The second and third rows of figure 18 present the phase-plane plots and the power spectral densities (PSDs) respectively, derived from large time intervals of the responses. For $U^{*} / U_{F}^{*}$ of 1.3 and 1.8, the plots in these two rows confirm the observations made from the corresponding $\alpha-t^{*}$ plots: the response is single-period and with increasing $U^{*} / U_{F}^{*}$, the oscillation amplitude increases and the frequency of oscillation decreases. For $U^{*} / U_{F}^{*}$ of 2.3 , the phase-plane plot is not a pure ellipse because of the LCO has multiple amplitudes. The PSD plot shows that there is a secondary frequency which has much smaller energy content than the primary frequency.

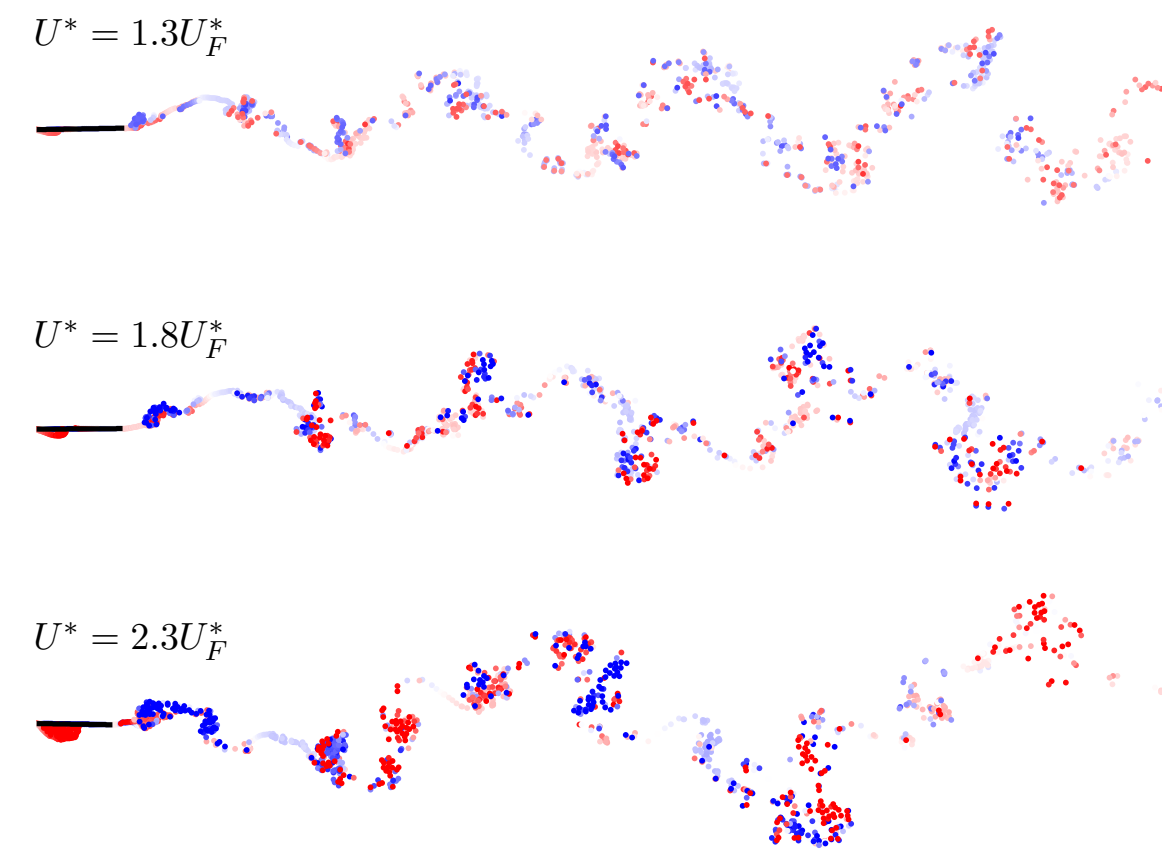

Figure 19: Parametric study A: flow topologies illustrated for three values of freestream velocity. From top to bottom: $U^{*}=1.3,1.8$ and $2.3 U_{F}^{*}$ respectively.

The flow topologies for the same three values of freestream velocity are 
shown in figure 19. The first two topologies are harmonic, with the $U^{*} / U_{F}^{*}=$ 1.8 case having larger accumulations of vorticity than than $U^{*} / U_{F}^{*}=1.3$ case. For $U^{*} / U_{F}^{*}=2.3$, the simulation shows a non-uniform wake structure with large transverse displacements of the vortical flow structures, which reflects the multiple-amplitude LCO seen for this velocity.

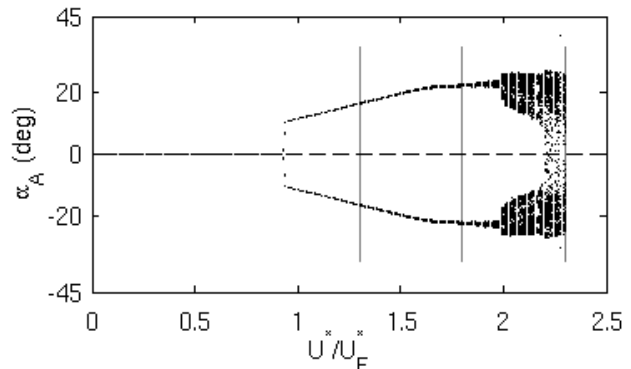

(a)

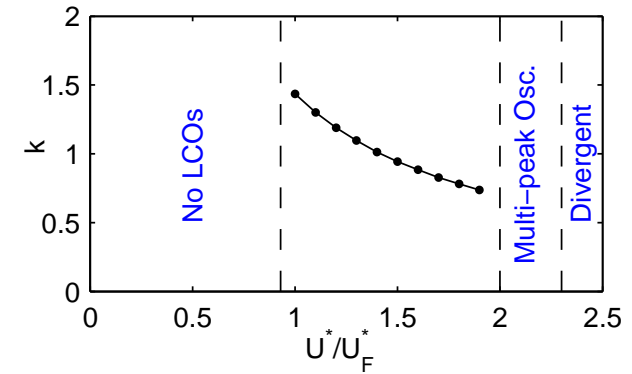

(c)

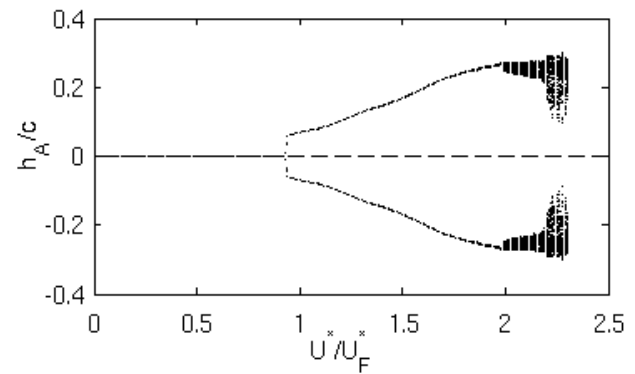

(b)

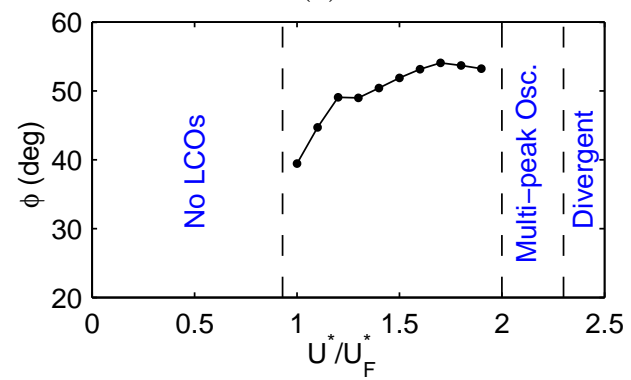

(d)

Figure 20: Parametric study A: bifurcation characteristics as a function of freestream velocity, for the parameters listed in table 1 with freestream velocity being variable. (a) Value of pitch angle $\alpha$ when $d \alpha / d t^{*}=0$, (b) value of plunge displacement $h / c$ when $d h / d t^{*}=0$, (c) reduced frequency of response in single-period regime, (d) phase angle between pitch and plunge in single-period regime.

Figures 20(a) and 20(b) show the variation in LCO characteristics over a large freestream-velocity range using bifurcation plots of pitch and plunge, while figures $20(\mathrm{c})$ and $20(\mathrm{~d})$ plot the variations of reduced frequency, $k$, and phase angle between pitch and plunge, $\phi$. On the vertical axis of figures 20(a), peak values in pitch during the LCO are plotted. For each value of $U^{*} / U_{F}^{*}$, the peak $\alpha$ values, identified by determining the instances at which $d \alpha / d t^{*}=0$, are plotted as was done in figure 18. In a similar manner, the peak values of the plunge oscillations at various $U^{*} / U_{F}^{*}$ are plotted in figure 20(b). The gray lines in 20(a) show the freestream velocity values used for the illustrations in figures 18 and 19. 
Figures 20(a) and (b) are seen to have a bifurcation at a value of $U^{*} / U_{F}^{*}$ slightly less than unity. While the bifurcation location in aeroelastic studies [16] is typically at $U^{*} / U_{F}^{*}=1$, the slight shift from unity here is because the $U_{F}^{*}$ is defined as the flutter velocity from Ref. [49], rather than as the velocity at which the bifurcation occurs.

When the freestream velocity is lower than the flutter velocity, the solution is stable and converges to zero amplitude for all initial displacements. For values of nondimensional freestream velocity between the flutter speed and approximately $2 U_{F}^{*}$, the bifurcation plots show single-period behavior. This transition from stable equilibrium to limit-cycle oscillation at the flutter speed appears to be a supercritical Hopf bifurcation [16].

The amplitude of single-period LCOs is seen to increase with increasing freestream velocity. At nondimensional velocities greater than $2 U_{F}^{*}$, departure from single-period behavior is seen. The peaks of the response take on multiple values and the oscillation appears to be quasiperiodic, as gleaned from the PSD plot in figure 18. This type of transition from periodic to quasiperiodic behavior has been reported, for instance, by Sarkar \& Bijl [30] in the case of a self-excited airfoil undergoing dynamic stall. Finally, divergent oscillations occur as the freestream velocity is increased beyond $2.3 U_{F}^{*}$. A detailed dynamical analysis of the response observed at high freestream velocities is beyond the scope of this paper. We simply refer to the oscillations seen between $2 U_{F}^{*}$ and $2.3 U_{F}^{*}$ as multi-peak oscillations. Figure 20(c) shows the variation of reduced frequency in the single-period-LCO regime. The reduced frequency has its highest value when the freestream velocity is close to the linear flutter velocity, and decreases with increasing freestream velocity. Figure 20(d) is a plot of the phase angle between pitch and plunge, with pitch leading plunge. The plot shows that the phase angle increases nonlinearly with increasing $U^{*} / U_{F}^{*}$. A study on the effect of varying initial conditions (not shown here) revealed that the LCOs resulting from intermittent LEV shedding are independent of initial conditions. This is in contrast with LCOs resulting from dynamic stall, which are dependent on the initial conditions [30].

It is noted that when the freestream velocity is varied, the Reynolds number also varies proportionally. Hence, the critical LESP value which depends on airfoil shape and Reynolds number would not be a constant over the range of freestream velocities, as assumed. Nevertheless, this assumption is still used for the purpose of illustrating the trends in LCO characteristics as a function of a single variable, the freestream velocity. The same caveat 
applies in section 4.4 where again, the freestream velocity is the parameter being varied.

\subsection{Parametric study B: Effect of cubic stiffening in torsional spring}

In this section, the added effect of positive cubic stiffening (hard spring) in torsion on the ensuing limit-cycle oscillations is analyzed. The base parameters listed in table 1 are employed, along with cubic stiffening in pitch of $\beta_{\alpha}=3$ (but keeping $\beta_{h}=0$ ). Considering freestream velocity as variable, bifurcation plots similar to those in section 4.3 are presented in figure 21 to study the LCO characteristics.
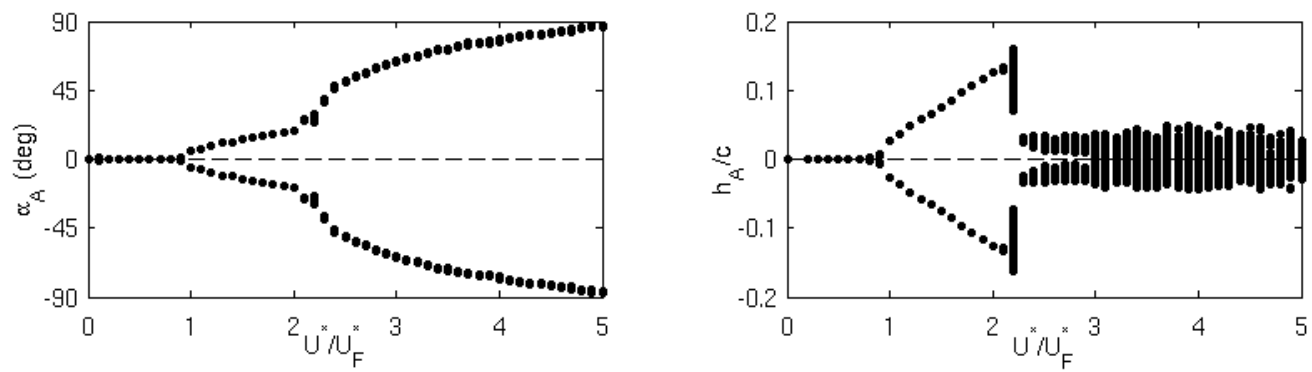

Figure 21: Parametric study B: bifurcation characteristics as a function of freestream velocity, for the parameters listed in table 1 and $\beta_{\alpha}=3$. Left: Value of pitch angle $\alpha$ when $d \alpha / d t^{*}=0$. Right: Value of plunge displacement $h / c$ when $d h / d t^{*}=0$.

Freestream velocities lower than the flutter velocity are seen to result in the response decaying to zero. This behavior was observed regardless of initial conditions. Between the flutter speed and $U^{*} \approx 2 U_{F}^{*}$, single-period limit-cycle behavior is observed. Up until this value of freestream velocity, the aeroelastic behavior is qualitatively similar to that without the cubic stiffening in section 4.3. For values of $U^{*}$ between $2 U_{F}^{*}$ and $2.3 U_{F}^{*}$, singleperiod response results whereas it was observed earlier that the response takes on multiple amplitudes in the absence of any structural nonlinearity. Finally, for values of dimensionless freestream velocity greater than $2.3 U_{F}^{*}$, singleperiod response in pitch along with small-amplitude multi-peak vibrations in plunge are seen. This is in contrast to the study without cubic stiffening, where divergence occurred at values of $U^{*}>2.3 U_{F}^{*}$. Thus, is it seen that one important effect of cubic hardening in high-frequency, vortex-dominated flows is to increase the range of stable, non-divergent LCOs.

The effect of cubic hardening on aeroelastic behavior (other than extending the range of stable LCOs) is studied by comparing single-period LCO 

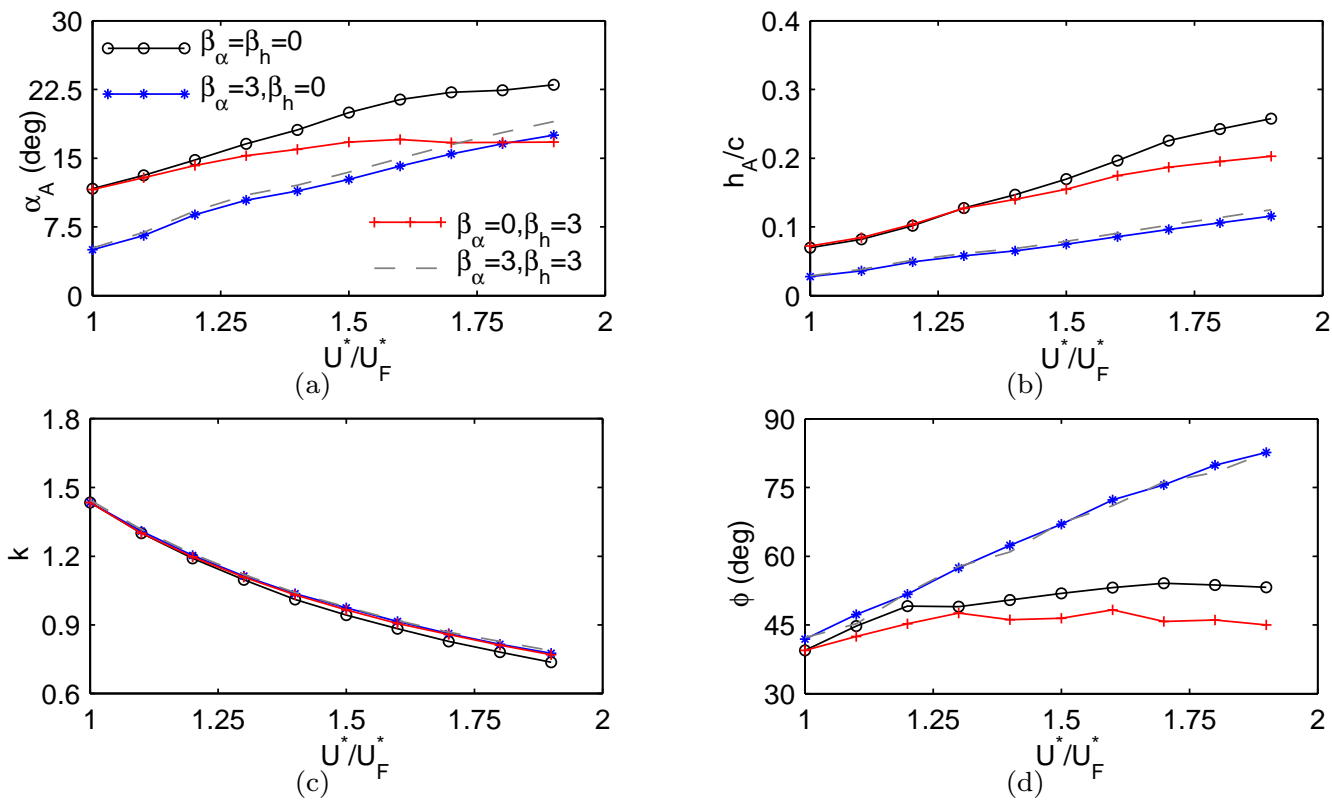

Figure 22: Parametric study B: comparison of LCO characteristics with and without cubic nonlinearity in pitch. (a) pitch angle, (b) plunge, (c) reduced frequency, (d) phase angle.

characteristics in the following cases: a) no cubic stiffening $\left(\beta_{\alpha}=\beta_{h}=0\right)$, (b) hard cubic stiffening in pitch only $\left(\beta_{\alpha}=3, \beta_{h}=0\right)$, (c) hard cubic stiffening in plunge only $\left(\beta_{\alpha}=0, \beta_{h}=3\right)$, and $(\mathrm{d})$ hard cubic stiffening in both pitch and plunge $\left(\beta_{\alpha}=\beta_{h}=3\right)$. LCO properties for values of freestream velocity between the flutter velocity and $1.9 U_{F}^{*}$ (where single-period LCOs exist in all studies) are compared in figure 22. Broadly, it is observed that in comparison with linear springs, the addition of any hard cubic stiffening results in lower pitch and plunge amplitudes. The reduced frequency of response is however, nearly the same for all cases. While a positive cubic stiffening in pitch increases the phase difference between pitch and plunge oscillation, the same cubic stiffening in plunge results in a decrease in the phase difference. For the values selected, stiffening in pitch has a more dominant impact on the aeroelastic behavior.

\subsection{Parametric study C: Effect of change in static unbalance and frequency ratio}

In this section, the variation in LCO characteristics with structural parameters is studied. Static unbalance $\left(x_{\alpha}\right)$ and frequency ratio $(\bar{\omega})$ are varied, 
and all other parameters are the same as those listed in table 1.

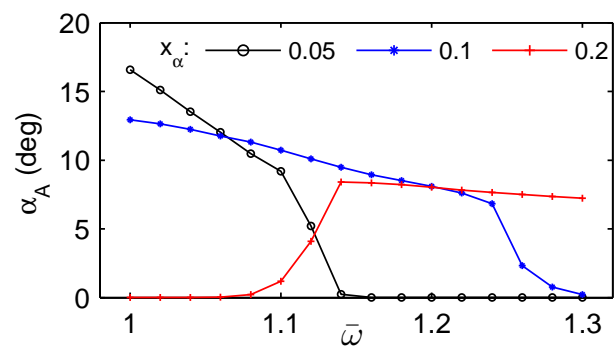

(a)

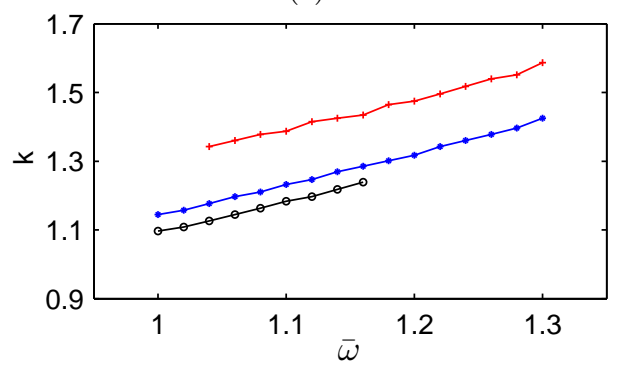

(c)

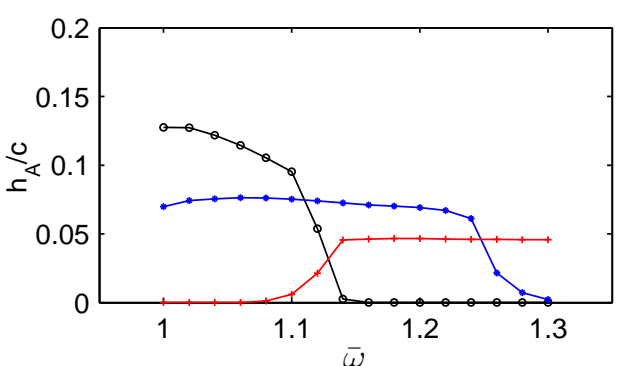

(b)

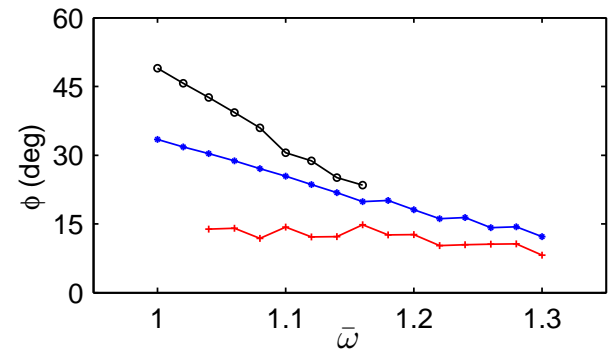

(d)

Figure 23: Parametric study B: LCO characteristics as function of structural parameters at a constant freestream velocity. The parameters listed in table 1 are used as a baseline with $x_{\alpha}$ and $\bar{\omega}$ as variables. (a) pitch amplitude, (b) plunge amplitude, (c) reduced frequency, (d) phase angle between pitch and plunge.

Figures 23(a) and (b) show the variation in pitch and plunge amplitudes of the resulting LCO over the parameter space identified from figure 14 . Three values of $x_{\alpha}(0.05,0.1,0.2)$ and frequency ratios between 1.0 and 1.3 are considered. The variation of LCO amplitudes in this space is seen to be very nonlinear. This is likely because the static unbalance and frequency ratio also affect the flutter speed, thereby making the relationship between the pitch/plunge amplitudes and these parameters very complex. It is noted that some of the amplitudes are zero valued. These cases correspond to simulations where the freestream velocity $\left(U^{*}=0.4667\right.$ from table 1$)$ is lower than the flutter velocity (which is a function of $x_{\alpha}$ and $\bar{\omega}$ ), thereby resulting in the response decaying to zero. Figures 23(c) and (d) are plots of reduced frequency and phase angle between pitch and plunge, for the LCOs in the chosen parameter space. The reduced frequency of response is seen to vary linearly with the frequency ratio $(\bar{\omega})$. Further, higher values of static unbalance (between 0.05 and 0.2 ), result in LCOs of higher frequency 
in pitch and plunge. It is noted again that this is the only study where the flutter velocity is also a function of the varying parameters, thereby resulting in a complex and counter-intuitive variations of pitch- and plungeLCO amplitudes.

\subsection{Parametric study D: Effect of change in airfoil shape (LESP $P_{\text {crit }}$ )}

The critical value of leading edge suction parameter (LESP) governs leading-edge vortex shedding in the aerodynamic model. This value is independent of motion kinematics, but depends on the airfoil shape and Reynolds number of operation. Since different airfoil-Re combinations may result in various critical LESP values, it is of interest to study the variation in LCO characteristics as a function of critical LESP. The parameters in table 1 are used as a baseline, with $L E S P_{\text {crit }}$ being a variable.

Limit-cycle properties (pitch and plunge amplitude, reduced frequency, phase angle) are plotted against critical LESP in figure 24. As the value of $L E S P_{\text {crit }}$ increases, pitch and plunge amplitudes increase linearly. It is interesting, however, that the reduced frequency of response is nearly the same value and independent of critical LESP. In earlier research, Ramesh et al. [51] have shown that airfoils with more rounded leading edges can support more suction, and hence have higher values of critical LESP. For example, a flat plate at $R e=1,000$ has $L E S P_{\text {crit }}=0.11$, and a NACA0015 airfoil at the same Reynolds number has $L E S P_{\text {crit }}=0.19$. Hence, it follows that more rounded airfoils result in LCOs of greater amplitudes but the same frequency. The increase in LCO amplitudes with critical LESP may be attributed to more a more rounded leading edge being able to sustain more leading-edge suction, thereby bounding the LCOs at a larger amplitude.

It is noted that a zero value of critical LESP corresponds to a perfectly sharp leading edge with continuous vortex shedding from the leading edge, and a "very high" critical LESP value $\left(L E S P_{\text {crit }}=5\right)$ models no vortex shedding from the leading edge. Hence, in the case of the latter, the aerodynamic model is "linear" and destructive oscillations would occur at speeds greater than the linear flutter speed, provided the structure is linear too. In the current study, the upper $L E S P_{\text {crit }}$ boundary is fixed at 0.3. As mentioned earlier, critical LESP values greater than this value are not considered because it is unrealistic that such a high suction could be attained without significant trailing-edge separation, which is not modeled here.

Flow topology plots at four equally spaced instants over one time period, for three values of critical LESP are shown in figure 25. Clearly, larger values 


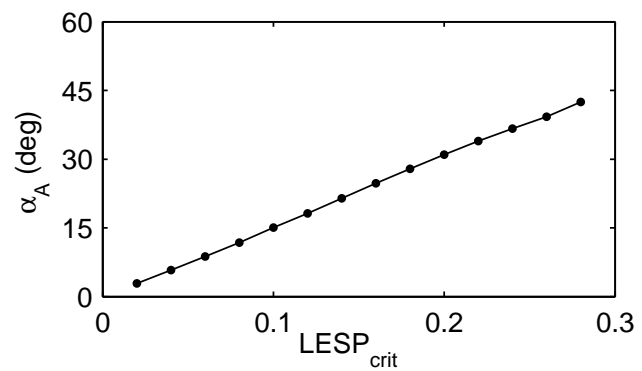

(a)

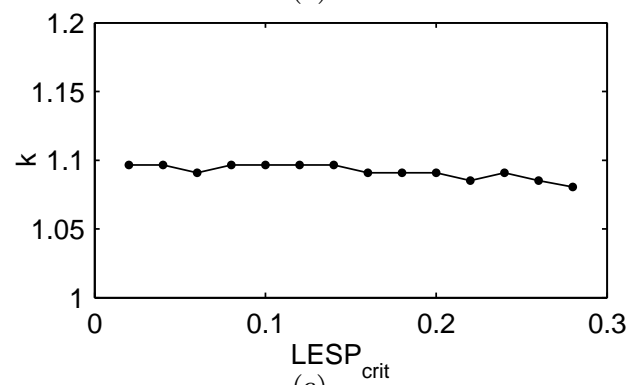

(c)

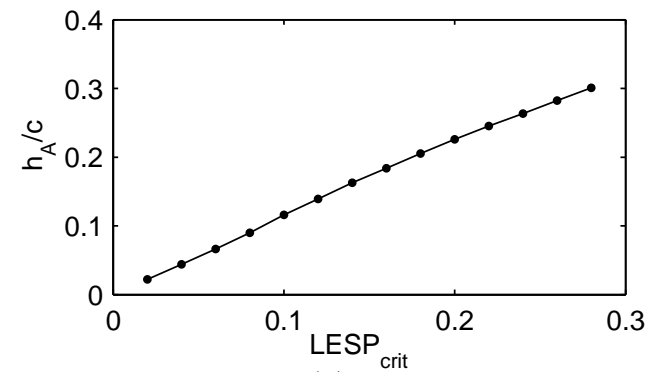

(b)

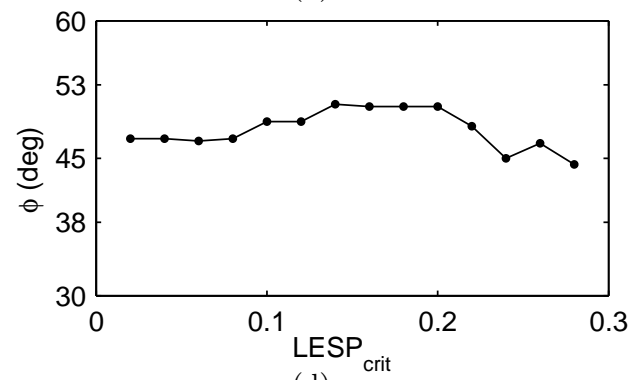

(d)

Figure 24: Parametric study D: LCO characteristics as function of critical LESP value. The parameters listed in table 1 are used as a baseline. (a) pitch amplitude, (b) plunge amplitude, (c) reduced frequency, (d)phase angle between pitch and plunge.

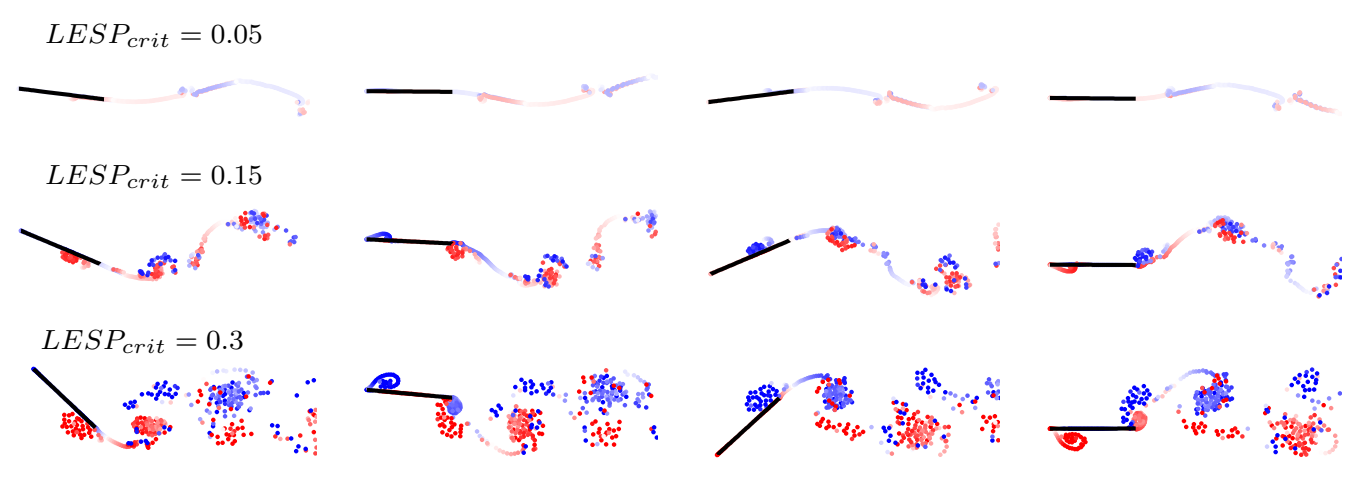
(a) $t^{*} / T^{*}=0$
(b) $t^{*} / T^{*}=0.25$
(c) $t^{*} / T^{*}=0.5$
(d) $t^{*} / T^{*}=0.75$

Figure 25: Parametric study D: flow topology plots for the limit-cycle responses obtained using different values of critical LESP. Plots (a)-(d) depict vorticity distributions at four equally spaced time intervals over one period of the oscillation.

of $L E S P_{\text {crit }}$ (more rounded airfoils) correspond to larger LCO amplitudes and larger vortical structures in the flowfield. The location of the LEV on the airfoil is nearly the same at any given time, regardless of $L E S P_{\text {crit }}$. 
This observation suggests that the frequency of vortex shedding and wake frequency are unchanged by the critical LESP, in accordance with the nearlyconstant LCO reduced frequency.

\section{Conclusions}

A geometrically-nonlinear 2-DOF airfoil model is combined with a discretevortex aerodynamic model having capability of intermittent leading-edge vortex shedding, to develop a nonlinear aeroelastic framework. This framework is used to investigate high-frequency limit-cycle oscillations at low Reynolds number where the unsteady aerodynamics are dominated by apparent-mass effects and leading-edge vortex shedding. A rounded flat-plate airfoil at a Reynolds number of 1,000 is the base configuration for the study. The discrete-vortex methodology used is computationally less expensive than CFD while still capturing the significant features of the aerodynamics, and is used to study the characteristics of LCOs in the regime under consideration and their dependence on various parameters.

For all freestream velocities lower than the airfoil's flutter velocity, the pitch and plunge responses converge to zero regardless of initial conditions. This is in contrast to studies at lower reduced frequencies (dynamic stall regime), where subcritical LCOs have been reported (e.g., in Ref. [30]). At velocities immediately greater than the flutter velocity, the airfoil's motion exhibits single-period limit-cycle behavior and the limiting values are independent of initial conditions for the cases considered. Hence, the bifurcation plot of LCO amplitude with freestream velocity as the bifurcation parameter exhibits a supercritical Hopf bifurcation at the flutter velocity. Beyond a certain velocity above the flutter velocity, single-period limit-cycle behavior is lost and multiple-peak oscillations are seen. At even higher velocities, the response is divergent when the structural model is linear. Divergence at these velocities may be avoided, and the envelope of stable LCOs increased, by using a spring with positive cubic stiffening (hard spring) rather than a linear spring. The parametric studies show that, in addition to structural nonlinearities, the vortex-dominated flowfield critically impacts the system dynamics leading to a complex and often hardly intuitive behavior.

The leading-edge suction parameter modulates LEV shedding in the discretevortex aerodynamic model used here. The critical LESP value depends on the airfoil shape and Reynolds number, and is a measure of the maximum suction force that the airfoil's leading edge can support without flow sepa- 
ration. Airfoils with more rounded leading edges therefore have a greater critical LESP value, and one with a sharp leading edge has a critical LESP of zero corresponding to the flow always being separated at the leading edge. The LCO characteristics as a function of critical LESP are studied to determine their dependence on airfoil shape. It is seen that, with increase in critical LESP, pitch and plunge amplitudes of the LCOs increase. It is very interesting, however, that the reduced frequency of oscillation is almost the same for all critical LESP values, suggesting that it is nearly independent of airfoil shape.

The parameter space was selected such that the resulting LCOs were of high reduced frequency, corresponding to values of frequency ratio close to unity. It is of interest that these values of frequency ratio also relate to low flutter velocities. While this is the very reason that such frequency ratios are undesirable in traditional aerospace applications, they may be beneficial for LCO-based power-harvesting mechanisms where low "cut-in" speeds are sought. Limit-cycle oscillations in this regime have not received much attention in the literature, and hence, additional studies are needed using higherorder computations and experiments, as well as dynamical-system analysis. In any case, the excellent accuracy/cost balance offered by the methodology presented in this paper suggests that it could be successfully employed to investigate optimum setups for power harvesting in the low-Reynolds-number regime. 


\section{Acknowledgments}

The authors thank the University Global Partnership Network (UGPN) who supported this collaboration between the University of Surrey and North Carolina State University through the grant titled "Harnessing fluid-structure interaction in wind power and sustainable air transport." The aerodynamic model was developed under a grant to the North Carolina State University from the U.S. Air Force Office of Scientific Research; grant number FA 9550-10-1-0120 monitored by program manager Dr. Douglas Smith. Their support is gratefully acknowledged. We thank Prof. Dumas for permission to use their flow plot from Ref. [26] in our figure 13.

[1] R. L. Bisplinghoff, H. Ashley, Aeroelasticity, Courier Dover Publications, 1996.

[2] Y. Fung, An introduction to the theory of aeroelasticity, Courier Dover Publications, 2002.

[3] M. Hamamoto, Y. Ohta, K. Hara, T. Hisada, Application of fluidstructure interaction analysis to flapping flight of insects with deformable wings, Advanced Robotics 21 (1-2) (2007) 1-21.

[4] T. Nakata, H. Liu, Aerodynamic performance of a hovering hawkmoth with flexible wings: a computational approach, Proceedings of the Royal Society B: Biological Sciences 279 (1729) (2012) 722-731.

[5] G. K. Taylor, M. S. Triantafyllou, C. Tropea, Animal locomotion, Springer-Verlag, 2010.

[6] E. Liani, S. Guo, G. Allegri, Aeroelastic effect on flapping wing performance, AIAA Paper 2007-2412 (2007).

[7] J. Tang, S. Chimakurthi, R. Palacios, C. E. S. Cesnik, W. Shyy, Computational fluid-structure interaction of a deformable flapping wing for micro air vehicle applications, AIAA Paper 2008-0615 (2008).

[8] W. Shyy, Y. Lian, J. Tang, H. Liu, P. Trizila, B. Stanford, L. Bernal, C. E. S. Cesnik, P. Friedmann, P. Ifju, Computational aerodynamics of low Reynolds number plunging, pitching and flexible wings for MAV applications, Acta Mechanica Sinica 24 (4) (2008) 351-373. 
[9] J. Young, J. Lai, M. F. Platzer, A review of progress and challenges in flapping foil power generation, Progress in Aerospace Sciences 67 (2014) $2-28$.

[10] M. Bryant, E. Garcia, Modeling and testing of a novel aeroelastic flutter energy harvester, Journal of Vibration and Acoustics 133 (1).

[11] J. A. Dunnmon, S. C. Stanton, B. P. Mann, E. H. Dowell, Power extraction from aeroelastic limit cycle oscillations, Journal of Fluids and Structures 27 (8) (2011) 1182-1198.

[12] L. Tang, M. P. Païdoussis, J. Jiang, Cantilevered flexible plates in axial flow: energy transfer and the concept of flutter-mill, Journal of Sound and Vibration 326 (1) (2009) 263-276.

[13] T. Kinsey, G. Dumas, G. Lalande, J. Ruel, A. Mehut, P. Viarouge, J. Lemay, Y. Jean, Prototype testing of a hydrokinetic turbine based on oscillating hydrofoils, Renewable Energy 36 (6) (2011) 1710-1718.

[14] T. Theodorsen, General theory of aerodynamic instability and the mechanism of flutter, NACA Rept. 496 (1935).

[15] T. Theodorsen, I. E. Garrick, Flutter calculations in three degrees of freedom, NACA Rept. 741 (1942).

[16] B. H. K. Lee, S. J. Price, Y. S. Wong, Nonlinear aeroelastic analysis of airfoils: bifurcation and chaos, Progress in Aerospace Sciences 35 (3) (1999) 205-334.

[17] B. H. K. Lee, L. Y. Jiang, Y. S. Wong, Flutter of an airfoil with a cubic restoring force, Journal of Fluids and Structures 13 (1) (1999) 75-101.

[18] S. J. Price, H. Alighanbari, B. H. K. Lee, The aeroelastic response of a two-dimensional airfoil with bilinear and cubic structural nonlinearities, Journal of Fluids and Structures 9 (2) (1995) 175-193.

[19] O. O. Bendiksen, Review of unsteady transonic aerodynamics: Theory and applications, Progress in Aerospace Sciences 47 (2) (2011) 135-167.

[20] J. G. Leishman, Principles of Helicopter Aerodynamics, Cambridge Aerospace Series, 2002. 
[21] C. P. Ellington, C. van den Berg, A. P. Willmott, A. L. R. Thomas, Leading-edge vortices in insect flight, Nature 384 (1) (1996) 626-630.

[22] W. Shyy, H. Liu, Flapping wings and aerodynamic lift: The role of leading-edge vortices, AIAA Journal 45 (12).

[23] C. P. Ellington, The novel aerodynamics of insect flight: applications to micro-air vehicles, Journal of Experimental Biology 202 (23) (1999) 3439-3448.

[24] M. H. Dickinson, K. G. Gotz, Unsteady aerodynamic performance of model wings at low Reynolds numbers, Journal of Experimental Biology 174 (1) (1993) 45-64.

[25] J. Anderson, K. Streitlien, D. Barrett, M. Triantafyllou, Oscillating Foils of High Propulsive Efficiency, Journal of Fluid Mechanics 360 (1) (1998) $41-72$.

[26] T. Kinsey, G. Dumas, Parametric study of an oscillating airfoil in a power-extraction regime, AIAA Journal 46 (6) (2008) 1318-1330.

[27] D. M. Tang, E. H. Dowell, Comparison of theory and experiment for non-linear flutter and stall response of a helicopter blade, Journal of Sound and Vibration 165 (2) (1993) 251-276.

[28] D. M. Tang, E. H. Dowell, Nonlinear aeroelasticity in rotorcraft, Mathematical and Computer modelling 18 (3) (1993) 157-184.

[29] C. T. Tran, D. Petot, Semi-empirical model for the dynamic stall of airfoils in view of the application to the calculation of responses of a helicopter blade in forward flight, Vertica 5 (1981) 35-53.

[30] S. Sarkar, H. Bijl, Nonlinear aeroelastic behavior of an oscillating airfoil during stall-induced vibration, Journal of Fluids and Structures 24 (6) (2008) 757-777.

[31] C. Chantharasenawong, Nonlinear aeroelastic behaviour of aerofoils under dynamic stall, Ph.D. thesis, Imperial College London (University of London) (2007).

[32] J. G. Leishman, T. S. Beddoes, A semi-empirical model for dynamic stall, Journal of the American Helicopter Society 34 (3) (1989) 3-17. 
[33] R. Kamakoti, W. Shyy, Fluid-structure interaction for aeroelastic applications, Progress in Aerospace Sciences 40 (8) (2004) 535-558.

[34] D. Poirel, V. Metivier, G. Dumas, Computational aeroelastic simulations of self-sustained pitch oscillations of a NACA0012 at transitional Reynolds numbers, Journal of Fluids and Structures 27 (8) (2011) 12621277.

[35] P. Sváček, M. Feistauer, J. Horáček, Numerical simulation of flow induced airfoil vibrations with large amplitudes, Journal of Fluids and Structures 23 (3) (2007) 391-411.

[36] Z. Peng, Q. Zhu, Energy harvesting through flow-induced oscillations of a foil, Physics of Fluids 21 (123602).

[37] K. D. Jones, M. F. Platzer, Time-domain analysis of low-speed airfoil flutter, AIAA Journal 34 (5) (1996) 1027-1033.

[38] K. Ramesh, A. Gopalarathnam, K. Granlund, M. V. Ol, J. R. Edwards, Discrete-vortex method with novel shedding criterion for unsteady airfoil flows with intermittent leading-edge vortex shedding, Journal of Fluid Mechanics 751 (2014) 500-538.

[39] K. Ramesh, A. Gopalarathnam, J. R. Edwards, M. V. Ol, K. Granlund, An unsteady airfoil theory applied to pitching motions validated against experiment and computation, Theoretical and Computational Fluid Dynamics 27 (6) (2013) 843-864.

[40] J. Katz, A. Plotkin, Low-Speed Aerodynamics, Cambridge Aerospace Series, 2000.

[41] I. E. Garrick, Propulsion of a flapping and oscillating airfoil, NACA Rept. 567 (1937).

[42] T. von Kármán, J. M. Burgers, General Aerodynamic Theory - Perfect Fluids, Vol. 2 of Aerodynamic theory: a general review of progress, Durand, W. F. , Dover Publications, 1963.

[43] J. Katz, Discrete vortex method for the non-steady separated flow over an airfoil, Journal of Fluid Mechanics 102 (1) (1981) 315-328. 
[44] G. H. Vatistas, V. Kozel, W. C. Mih, A simpler model for concentrated vortices, Experiments in Fluids 11 (1) (1991) 73-76.

[45] O. H. Hald, Convergence of vortex methods for Euler's equations, II, SIAM Journal on Numerical Analysis 16 (5) (1979) 726-755.

[46] A. Leonard, Vortex methods for flow simulation, Journal of Computational Physics 37 (3) (1980) 289-335.

[47] J. Carrier, L. Greengard, V. Rokhlin, A fast adaptive multipole algorithm for particle simulations, SIAM Journal on Scientific and Statistical Computing 9 (4) (1988) 669-686.

[48] J. C. Butcher, Numerical Methods for Ordinary Differential Equations, 2nd Edition, John Wiley \& Sons, Ltd, Chichester, UK, 2008.

[49] J. Murua, R. Palacios, J. Peiró, Camber effects in the dynamic aeroelasticity of compliant airfoils, Journal of Fluids and Structures 26 (4) (2010) 527-543.

[50] FLUENT, Software Package, Ver. 6.1, ANSYS, Inc., Lebanon, NH, 2003.

[51] K. Ramesh, J. Ke, A. Gopalarathnam, J. R. Edwards, Effect of airfoil shape and Reynolds number on leading edge vortex shedding in unsteady flows, AIAA Paper 2012-3025 (2012). 